KCL-MTH-10-02

\title{
The very-extended trombone
}

\author{
Fabio Riccioni \\ Department of Mathematics \\ King's College London \\ Strand London WC2R $2 L S$ \\ $U K$ \\ E-mail: Fabio.Riccioni@kcl.ac.uk
}

\begin{abstract}
Starting from the very-extended Kac-Moody algebra $E_{11}$, we consider the algebra $E_{11, D}^{\text {local }}$, obtained by adding to the non-negative level $E_{11}$ generators the $D$-dimensional momentum operator and an infinite set of additional generators that promote the global $E_{11}$ symmetries to gauge ones. We determine all the possible trombone deformations of this algebra, that is the deformations that involve the $D$-dimensional scaling operator. The Jacobi identities imply that such deformations are uniquely determined by a single tensor belonging to the same representation of the internal symmetry group as the vector generators and satisfying additional quadratic constraints. The non-linear realisation of the deformed algebra gives the field strengths of the theory which are those of any possible maximal supergravity theory in which the global scaling symmetry is gauged in any dimension. All the possible deformed algebras are in one to one correspondence with all such maximal supergravity theories. The tensor that parametrises the deformation is identified with the embedding tensor that is used to parametrise all maximal supergravity theories with gauged scaling symmetry, and the quadratic constraints that we determine exactly coincide with the field theory results.
\end{abstract}




\section{Introduction}

The hidden symmetries of ungauged maximal supergravities have played a crucial role in our understanding of string theory dualities. These theories in dimension lower than ten are unique and can be obtained by torus dimensional reduction from both eleven dimensional [1] and IIB [2] supergravities. For instance, in four dimensions the theory develops an $E_{7(7)}$ symmetry [3], while the hidden symmetry of the five-dimensional theory is $E_{6(6)}$ [4]. In general we refer to the internal symmetry group of the ungauged maximal $D$-dimensional supergravity theory as the Cremmer-Julia group, and we denote it by $E_{11-D(11-D)}$.

Gauged maximal supergravities generally arise as deformations of the ungauged ones by imposing supersymmetry together with gauge invariance with respect to a subgroup of $E_{11-D(11-D)}$. For instance the four dimensional $\mathcal{N}=8$ theory of [5] is a deformation of the massless maximal supergravity of [3] where an $S O(8)$ subgroup of $E_{7(7)}$ is gauged. Relatively recently, all maximal gauged supergravity theories in each dimension $D$ have been classified in terms of a single object called the embedding tensor which can be thought of as belonging to a representation of the internal symmetry group $E_{11-D(11-D)}$ of the supergravity theory in $D$ dimensions $[6,7,8,9,10,11,12]$. Supersymmetry specifies the $E_{11-D}$ representation of the embedding tensor by imposing a set of linear (or representation) constraints, while imposing that the embedding tensor is a constant breaks the $E_{11-D}$ symmetry to the subgroup the embedding tensor is an invariant tensor of. This subgroup is indeed the gauge group, and consistency imposes additional quadratic constraints on the embedding tensor, which can be viewed as the Jacobi identities for the structure constants of this gauge group.

Maximal supergravity theories have a very elegant classification in terms of the veryextended infinite-dimensional Kac-Moody algebra $E_{11}$ [13]. This algebra was first conjectured in [13] to be a symmetry of M-theory. The maximal supergravity theory in $D$ dimensions corresponds to decomposing $E_{11}$ in terms of $G L(D, \mathbb{R}) \otimes E_{11-D}$, and thus the occurrence of the internal symmetry $E_{11-D}$ appears natural from this perspective. The $G L(D, \mathbb{R})$ group corresponds to the constant part of the diffeomorphism group in $D$ dimensions, and gravity is described in this framework as the non-linear realisation of the diffeomorphism group with the $D$ dimensional Lorentz group as local subgroup $[14,15,16]$. For instance, the IIA theory naturally has from the $E_{11}$ viewpoint an $\mathbb{R}^{+}$symmetry corresponding to the shift of the dilaton. Decomposing the adjoint representation of $E_{11}$ with 
respect to the subalgebra associated to the IIA theory one obtains generators that are associated to the IIA fields and their duals [13]. One also finds a generator with nine antisymmetric ten-dimensional spacetime indices, which is associated to a 9-form [17]. This 9-form has a 10-form field strength, which can be thought as the dual of the mass parameter of Romans. Therefore the Romans massive IIA is naturally encoded in $E_{11}$ [18].

More generally, decomposing the $E_{11}$ algebra in a given dimension and considering only the level zero generators (that is the generators of $G L(D, \mathbb{R}) \otimes E_{11-D}$, that are associated to the graviton and the scalars) and the positive level generators with completely antisymmetric indices, that are associated to forms, one finds in all cases the field content of the $D$-dimensional supergravity theory, in a democratic formulation in which all fields appear together with their magnetic duals $[17,19]$. One also finds $D-1$ form generators in representations of $E_{11-D}$ which remarkably are the same as those fixed by the linear constraints on the embedding tensor for the maximal gauged supergravities in dimension $D[19,20]$. Exactly like in the case on Romans, one thinks of the $D-1$ form fields as being dual to the embedding tensor, obtaining in this way a classification of all possible maximal gauged supergravities in terms of $E_{11}$.

The action of positive level $E_{11}$ generators with completely antisymmetric spacetime indices in the non-linear realisation corresponds to gauge transformations for the associated form fields that are linear in the spacetime coordinates. In [21] this algebra was enlarged in order to include arbitrary gauge transformations. The resulting algebra includes the nonnegative level generators as well as momentum and an infinite set of additional generators, that were called Ogievetsky, or Og generators, that correspond to an expansion in the spacetime coordinates of the gauge parameters. This extension is dimension-dependent, and it was called $E_{11, D}^{\text {local }}$ in [21]. From the non-linear realisation of the $E_{11, D}^{\text {local }}$ algebra with as local subalgebra the $D$ dimensional Lorentz algebra times the maximal compact subalgebra of $E_{11-D}$ one computes all the field strengths of the massless maximal supergravity in $D$ dimensions.

Given the local $E_{11}$ algebra in $D$ dimensions, one can consider its massive deformations. In [22] the deformations that do not involve the $G L(D, \mathbb{R})$ generators were studied, and the consistency of the deformed algebra implies that all possible deformations are parametrised by a constant quantity that turns out to be the embedding tensor. Remarkably, the Jacobi identities impose constraints on the embedding tensor that are exactly the linear and quadratic constraints that were obtained in the field theory analysis. All the 
possible deformations are thus in one to one correspondence with all the possible gauged supergravities resulting from the gauging of a subgroup of $E_{11-D}$, while the Maurer-Cartan form gives all the field strengths in a straightforward way.

There are maximal gauged supergravities that do not arise from the gauging of a subgroup of the internal symmetry, but correspond to the gauging of the global scaling symmetry, also called "trombone" symmetry, that leaves the field equations invariant, but rescales the action. The fact that the scaling symmetry is not a symmetry of the lagrangian implies that the corresponding gauged theory does not admit a lagrangian formulation, but only field equations. The first example of such a theory is the gauged IIA theory of [23, 24], while the nine-dimensional analogue was discussed in [25]. Recently, in [26] a systematic classification of these theories in dimension from three to six was provided. This was achieved by the introduction of a new type of embedding tensor, belonging to the $E_{11-D}$ representation which is conjugate to the one of the vector fields. The consistency of the gauge algebra implies additional quadratic constraints for this embedding tensor.

In [27] it was shown that the local $E_{11}$ algebra admits a new type of deformations that are associated to maximal supergravities in which the trombone symmetry is gauged. These deformations involve the generator of scale transformations, which is the trace of the $G L(D, \mathbb{R})$ generators. This deformation was discussed in detail for the IIA case, corresponding to the gauged IIA theory of $[23,24]$.

In this paper we perform a systematic analysis of these deformations in any dimension from three to nine. In particular, we show that the deformations are parametrised by a constant quantity in the same $E_{11-D}$ representation as the vector generator, which is identified with the embedding tensor of [26]. We show that the Jacobi identities imply quadratic constraints for the embedding tensor that are exactly equivalent to those derived in [26] for the cases from three to six dimensions, and we also derive the quadratic constraints in seven, eight and nine dimensions. Moreover, from the non-linear realisation we derive the field strengths and gauge transformations of the fields.

It is important to stress that the local $E_{11}$ algebra is not compatible with the full $E_{11}$ symmetry, and only its $G L(D, \mathbb{R}) \otimes E_{11-D}$ subalgebra survives the introduction of momentum and the $\mathrm{Og}$ generators in $D$ dimensions. A similar argument applies to all the deformed cases, where $E_{11-D}$ is further broken by the embedding tensor to the gauge subgroup. $E_{11}$ is considered throughout this paper as the universal algebraic framework to describe the gauge algebra (including gravity) of all maximal supergravity theories. 
The paper is organised as follows. In section 2 we derive the general method of constructing the local $E_{11}$ algebra associated to the trombone deformations in any dimension. In section $D$, with $D=3, \ldots, 9$, we explicitly derive the deformed algebra in a given dimension $D$. Section 10 contains the conclusions.

\section{The general method}

In this section we perform a general analysis which will then be used from sections 3 to 9 in each dimension separately. The analysis is based on the results of [27], where it was shown how one can deform the local $E_{11}$ algebra corresponding to IIA supergravity to obtain a non-linear realisation corresponding to the gauged IIA theory of [23, 24].

In [22] it was shown that all gauged supergravities that result from the gauging of a subgroup of the internal symmetry group arise from all possible deformations of the local $E_{11}$ algebra in $D$ dimensions that do not involve the $G L(D, \mathbb{R})$ generators. In order to show this, in section 2 of [22] a general notation was introduced to discuss in a single framework all different dimensions. In this paper we consider in any dimension deformations that also involve the $G L(D, \mathbb{R})$ generators, and in this section we develop a general formalism which is the analogue for these deformations to section 2 of [22]. In particular all the notations are taken from there. We thus decompose $E_{11}$ in terms of $G L(D, \mathbb{R}) \otimes E_{11-D}$. This corresponds to deleting node $D$ in the $E_{11}$ Dynkin diagram of fig. 1 . In the decomposition

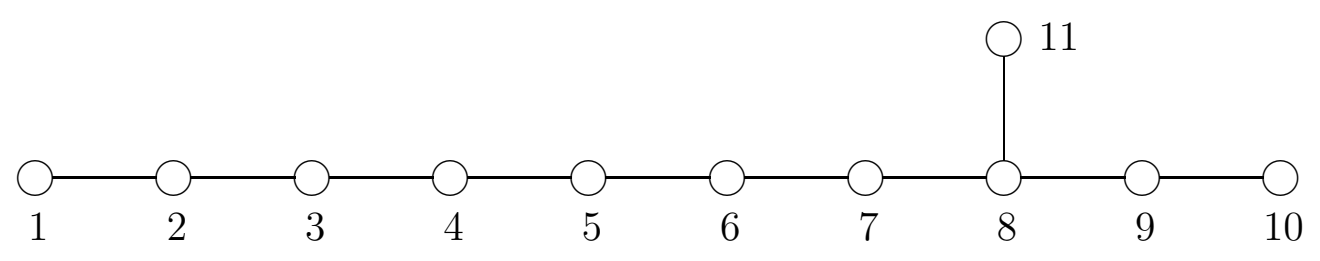

Figure 1: The $E_{11}$ Dynkin diagram.

of the adjoint representation of $E_{11}$ in terms of representations of $G L(D, \mathbb{R}) \otimes E_{11-D}$, we are only interested in the level zero generators, that is the generators of $G L(D, \mathbb{R})$ and those of $E_{11-D}$, and the positive level generators with completely antisymmetric $G L(D, \mathbb{R})$ indices. These are

$$
K^{\mu}{ }_{\nu} \quad R^{\alpha} \quad R^{\mu_{1}, M_{1}} \quad R^{\mu_{1} \mu_{2}, M_{2}} \quad \ldots \quad .
$$

The generator $R^{\mu_{1} \ldots \mu_{n}, M_{n}}$ carries the representation $\mathbf{R}_{\mathbf{n}}$ of $E_{11-D}$ which transforms the $M_{n}$ 
index. The $E_{11-D}$ generators in such representation are $D_{N_{m}}^{\alpha}{ }^{M_{m}}$. The $E_{11}$ algebra involving the form generators is

$$
\left[R^{\mu_{1} \ldots \mu_{m}, M_{m}}, R^{\nu_{1} \ldots \nu_{n}, N_{n}}\right]=f^{M_{m} N_{n}}{ }_{P_{m+n}} R^{\mu_{1} \ldots \mu_{m} \nu_{1} \ldots \nu_{n}, P_{m+n}}
$$

and

$$
\left[R^{\alpha}, R^{\mu_{1} \ldots \mu_{m}, M_{m}}\right]=\left(D^{\alpha}\right)_{N_{m}}{ }^{M_{m}} R^{\mu_{1} \ldots \mu_{m}, N_{m}} \quad, \quad\left[R^{\alpha}, R^{\beta}\right]=f_{\gamma}^{\alpha \beta} R^{\gamma},
$$

where $f^{\alpha \beta}{ }_{\gamma}$ are the structure constants of $E_{11-D}$ and $f^{M_{m} N_{n}}{ }_{P_{n+m}}$ are generalised structure constants.

While the scalar generators are associated to the global symmetry $E_{11-D}$, all the form generators are associated to gauge fields, and as explained in reference [21], the global symmetry associated to the form generators is promoted to a local one by the addition of the spacetime translation operator $P_{\mu}$ and an infinite number of so called Ogievetsky, or Og, generators. In fact for our purposes we need only add the lowest order, or Og 1, such generators, $K^{\mu, \nu_{1} \ldots \nu_{n}, M_{n}}$, which by definition obey the commutator

$$
\left[K^{\mu, \nu_{1} \ldots \nu_{n}, M_{n}}, P_{\rho}\right]=\delta_{\rho}^{\mu} R^{\nu_{1} \ldots \nu_{n}, M_{n}}-\delta_{\rho}^{[\mu} R^{\left.\nu_{1} \ldots \nu_{n}\right], M_{n}}
$$

Each generator $K^{\mu, \nu_{1} \ldots \nu_{n}, M_{n}}$ is associated with the $E_{11}$ generator $R^{\mu_{1} \ldots \mu_{n}, M_{n}}$ and carries the same internal symmetry representation, $\mathbf{R}_{\mathbf{n}}$. It also satisfies $K^{\left[\mu, \nu_{1} \ldots \nu_{n}\right], M_{n}}=0$.

The deformations of the above $E_{11, D}^{\text {local }}$ algebra considered in [22] are associated to gaugings of subgroups of the internal symmetry group $E_{11-D}$. As such, they do not involve the $G L(D, \mathbb{R})$ generators, which commute with the $E_{11-D}$ generators. Correspondingly, these deformations leave the $D$ dimensional metric invariant. Here we want to consider a new class of deformations, and we take as our starting point the deformed commutator

$$
\left[R^{\mu, M_{1}}, P_{\nu}\right]=\delta_{\nu}^{\mu}\left(\Theta^{M_{1}} K+a \Theta^{N_{1}} g_{\alpha \beta} D_{N_{1}}^{\alpha M_{1}} R^{\beta}\right)
$$

where the parameter $a$ is to be determined, and we denote with $K$ the trace of the $G L(D, \mathbb{R})$ generators $K^{\mu}{ }_{\nu}$

$$
K=K_{\mu}^{\mu}
$$

whose commutator with the form generators is

$$
\left[K, R^{\mu_{1} \ldots \mu_{m}, M_{m}}\right]=m R^{\mu_{1} \ldots \mu_{m}, M_{m}} .
$$

It can be shown that an additional term of the form

$$
\left[R^{\mu, M_{1}}, P_{\nu}\right]=\Theta^{M_{1}} K^{\mu}{ }_{\nu}
$$


which involves the $G L(D, \mathbb{R})$ generator and not just its trace, can be reabsorbed in a redefinition of $R^{\mu, M_{1}}$ of the form

$$
R^{\mu, M_{1}} \rightarrow R^{\mu, M_{1}}-2 \Theta^{M_{1}} K_{\nu}^{\mu \nu}
$$

where $K_{\rho}^{\mu \nu}$ is the Og 1 gravity generator. Therefore, we will not consider this term in this paper.

These deformations are therefore characterised by an embedding tensor in the same representation $\mathbf{R}_{\mathbf{1}}$ as the 1 -form generators. The consistency of the algebra will then in general imply that the commutator of the $n$-form generator with momentum does not vanish, and we thus write

$$
\left[R^{\mu_{1} \ldots \mu_{n}, M_{n}}, P_{\nu}\right]=\Theta^{M_{1}} S^{M_{n}} M_{1} M_{n-1} \delta_{\nu}^{\left[\mu_{1}\right.} R^{\left.\mu_{2} \ldots \mu_{n}\right], M_{n-1}}
$$

where $S^{M_{n}}{ }_{M_{1} M_{n-1}}$ is an invariant tensor of $E_{11-D}$. This defines the trombone deformation of the algebra $E_{11, D}^{\text {local }}$. The closure of the algebra also implies that the commutators of eqs. (2.2) and (2.4) become

$$
\begin{gathered}
{\left[R^{\mu_{1} \ldots \mu_{m}, M_{m}}, R^{\nu_{1} \ldots \nu_{n}, N_{n}}\right]=f^{M_{m} N_{n}}{ }_{P_{m+n}} R^{\mu_{1} \ldots \mu_{m} \nu_{1} \ldots \nu_{n}, P_{m+n}}} \\
+\Theta^{M_{1}} T^{M_{m} N_{n}}{ }_{M_{1} M_{m+n-1}} K^{\left[\mu_{1}, \mu_{2} \ldots \mu_{n}\right] \nu_{1} \ldots \nu_{m}, M_{m+n-1}} \\
{\left[K^{\mu, \nu_{1} \ldots \nu_{m}, M_{m}}, P_{\rho}\right]=\delta_{\rho}^{\mu} R^{\nu_{1} \ldots \nu_{m}, M_{m}}-\delta_{\rho}^{[\mu} R^{\left.\nu_{1} \ldots \nu_{m}\right], M_{m}}} \\
+\Theta^{N_{1}} U^{M_{m}} N_{1} P_{m-1} \delta_{\rho}^{\left[\nu_{1}\right.} K^{\left.|\mu,| \nu_{2} \ldots \nu_{m}\right], P_{m-1}}
\end{gathered}
$$

where $T^{M_{m} N_{n}} M_{1} M_{m+n-1}$ and $U^{M_{m}}{ }_{N_{1} P_{m-1}}$ are invariant tensors of $E_{11-D}$.

The tensor $\Theta^{M_{1}}$ is clearly not an invariant tensor of $E_{11-D}$, given that it transforms in the $\mathbf{R}_{\mathbf{1}}$ representation. Therefore assuming that the embedding tensor $\Theta^{M_{1}}$ is constant breaks the $E_{11-D}$ symmetry. For this to be consistent, $\Theta^{M_{1}}$ must satisfy quadratic constraints. Starting from the algebra of eqs. (2.5), (2.10) and (2.11), as well as eq. (2.3), and imposing the closure of the Jacobi identities, we now determine the conditions that the invariant tensors $S^{M_{n}} M_{1} M_{n-1}, T^{M_{m} N_{n}} M_{1} M_{m+n-1}$ and $U^{M_{m}} N_{1} P_{m-1}$ must satisfy, as well as the quadratic constraints on the embedding tensor $\Theta^{M_{1}}$. As we will see in sections from 3 to 6 , these constraints are exactly the constraints determined in [26] from a field theory analysis in dimensions from 3 to 6 . In sections 7,8 and 9 we will also determine explicitly the constraints in dimensions 7,8 and 9. The ten-dimensional case, corresponding to the gauged IIA theory of [23, 24], was analysed in [27], where it was also shown how the ten-dimensional deformed algebra arises from an eleven-dimensional perspective. 
We first consider the Jacobi identity involving the operators $R^{\mu, M_{1}}, R^{\nu, N_{1}}$ and $P_{\rho}$. We get two conditions, coming from the antisymmetric and symmetric terms in $\mu \nu$ respectively, which are

$$
\begin{aligned}
& f^{M_{1} N_{1}}{ }_{P_{2}} S^{P_{2}}{ }_{Q_{1} P_{1}}=2\left[\delta_{Q_{1}}^{\left(M_{1}\right.} \delta_{P_{1}}^{\left.N_{1}\right)}+a D_{\alpha Q_{1}}{ }^{\left(M_{1}\right.} D_{P_{1}}^{\left.\alpha N_{1}\right)}\right] \\
& T^{M_{1} N_{1}}{ }_{Q_{1} P_{1}}=2\left[\delta_{Q_{1}}^{\left[M_{1}\right.} \delta_{P_{1}}^{\left.N_{1}\right]}+a D_{\alpha Q_{1}}{ }^{\left[M_{1}\right.} D_{P_{1}}^{\left.\alpha{ }^{N_{1}}\right]}\right] .
\end{aligned}
$$

These two conditions determine $S^{P_{2}} Q_{1} P_{1}$ and $T^{M_{1} N_{1}}{ }_{Q_{1} P_{1}}$, and as we will see in all cases in the next sections, they also determine independently the parameter $a$.

Proceeding this way one determines all the conditions that the invariant tensors in eqs. (2.10) and (2.11) satisfy. Given that our goal will be the computation of the field strengths and gauge transformations of the fields, we are only interested in the part of the algebra that involves the deformed $E_{11}$ generators. Therefore we will from now on neglect the contribution to the Jacobi identities coming from the Og generators, and always assume that the upstairs spacetime indices are completely antisymmetrised. This is exactly the same attitude that was taken in [22] when computing the field strengths and gauge transformations for the deformed algebras associated to gauged supergravities in which an internal symmetry is gauged. Considering only the antisymmetric contribution, the Jacobi identity involving $R^{\mu, M_{1}}, R^{\nu_{1} \ldots \nu_{n}, N_{n}}$ and $P_{\rho}$ gives

$$
n \delta_{P_{1}}^{M_{1}} \delta_{R_{n}}^{N_{n}}+a D_{\alpha, P_{1}}^{M_{1}} D_{R_{n}}^{\alpha N_{n}}=S^{N_{n}}{ }_{P_{1} Q_{n-1}} f^{M_{1} Q_{n-1}}{ }_{R_{n}}+f^{M_{1} N_{n}}{ }_{S_{n}} S^{S_{n+1}}{ }_{P_{1} R_{n}}
$$

Starting from the first of eqs. (2.12), which determines $S^{P_{2}} Q_{1} P_{1}$, this equation determines by induction all the invariant tensors $S^{N_{n}} P_{1} Q_{n-1}$ in eq. (2.10). This is all we need to compute the field strengths and gauge transformations of the fields. Nonetheless, it is important to stress that the consistency of the algebra imposes in general the presence of the $\mathrm{Og}$ generators in the first of eqs. (2.11), and the invariant tensors $T^{M_{m} N_{n}} M_{1} M_{m+n-1}$ and $U^{M_{m}} N_{1} P_{m-1}$ can easily be determined requiring the closure of the Jacobi identities and considering the terms which are not completely antisymmetric in their spacetime indices.

We then consider the quadratic constraints. These arise from Jacobi identities involving a deformed $E_{11}$ generator and two momenta, or a deformed $E_{11}$ generator, the momentum operator and the scalar operator $\Theta^{M_{1}} K+a \Theta^{N_{1}} D_{\alpha, N_{1}}{ }^{M_{1}} R^{\alpha}$. The Jacobi identity involving the vector generator $R^{\mu, M_{1}}$ and two momenta imposes that

$$
\left[\Theta^{M_{1}} K+a \Theta^{N_{1}} D_{\alpha, N_{1}}^{M_{1}} R^{\alpha}, P_{\mu}\right]=0
$$


This implies that the deformed commutation relation

$$
\left[\Theta^{N_{1}} D_{\alpha, N_{1}}^{M_{1}} R^{\alpha}, P_{\mu}\right]=\frac{1}{a} \Theta^{M_{1}} P_{\mu}
$$

holds. The Jacobi identity involving $R^{\mu_{1} \mu_{2}, M_{2}}$ and two momentum operators gives

$$
\Theta^{M_{1}} \Theta^{N_{1}} S^{M_{2}}{ }_{M_{1} N_{1}} K+a \Theta^{M_{1}} \Theta^{P_{1}} S^{M_{2}}{ }_{M_{1} N_{1}} D_{\alpha, P_{1}}{ }^{N_{1}} R^{\alpha}=0
$$

The term proportional to $K$ vanishes provided that $\Theta^{M_{1}}$ satisfies the constraint

$$
\Theta^{M_{1}} \Theta^{N_{1}} S^{M_{2}} M_{1} N_{1}=0
$$

which is precisely the generalised "pure spinor constraint" found in [26] in $D=3,4,5,6$. Using the invariance of $S^{M_{2}}{ }_{M_{1} N_{1}}$, this constraint means that the product of two embedding tensors vanishes when projected on the representation $\mathbf{R}_{\mathbf{2}}$ to which the 2 -form generators belong. The term proportional to $R^{\alpha}$ also vanishes using eq. (2.17) and the invariance of $S^{M_{2}} M_{1} N_{1}$. The Jacobi identity involving $R^{\mu_{1} \ldots \mu_{n}, M_{n}}$, with $n>1$, and two momentum operators gives

$$
\Theta^{N_{1}} \Theta^{P_{1}} S^{M_{n}}{ }_{N_{1} Q_{n-1}} S^{Q_{n-1}}{ }_{P_{1} R_{n-2}}=0
$$

As will be clear in the next sections, this constraint derives in all cases from eq. (2.17) and the condition of $E_{11-D}$ invariance of $S^{M_{n}} N_{1} Q_{n-1}$.

We now consider the quadratic constraints resulting from the Jacobi identity involving a deformed $E_{11}$ generator, the momentum operator and the scalar operator $\Theta^{M_{1}} K+$ $a \Theta^{N_{1}} D_{\alpha, N_{1}}^{M_{1}} R^{\alpha}$. We first consider the case in which the $E_{11}$ generator is $R^{\mu, M_{1}}$, and we get the equation

$$
\begin{aligned}
& {\left[\Theta^{M_{1}} \Theta^{P_{1}}+a \Theta^{N_{1}} \Theta^{Q_{1}} D_{\alpha, N_{1}}{ }^{M_{1}} D_{Q_{1}}^{\alpha{ }^{P_{1}}}\right] K} \\
& +a\left[\Theta^{M_{1}} \Theta^{Q_{1}} D_{\alpha, Q_{1}}{ }^{P_{1}}+a \Theta^{N_{1}} \Theta^{S_{1}} D_{\beta, N_{1}}{ }^{M_{1}} D_{S_{1}}^{\beta^{Q_{1}}} D_{\alpha, Q_{1}}{ }^{P_{1}}\right] R^{\alpha}=0 .
\end{aligned}
$$

Both the $K$ and the $R^{\beta}$ term give the condition

$$
\Theta^{N_{1}} \Theta^{Q_{1}}\left[\delta_{N_{1}}^{M_{1}} \delta_{Q_{1}}^{P_{1}}+a D_{\alpha, N_{1}}^{M_{1}} D_{Q_{1}}^{\alpha{ }^{P_{1}}}\right]=0
$$

As we have already mentioned, the parameter $a$ is determined by imposing the closure of the Jacobi identity involving two 1-form generators and the momentum operator. We will show in the next sections that using this value of $a$, eq. (2.20) is automatically implied by eq. (2.17) in all dimensions. 
The last set of quadratic constraints come from the Jacobi identity involving $R^{\mu_{1} \ldots \mu_{m}, M_{m}}$, momentum and the scalar operator. This gives

$$
\begin{aligned}
& \Theta^{N_{1}} \Theta^{Q_{1}}\left[\delta_{N_{1}}^{M_{1}} S^{P_{n}}{ }_{Q_{1} R_{n-1}}-a S^{P_{n}}{ }_{Q_{1} Q_{n-1}} D_{\alpha, N_{1}}{ }^{M_{1}} D_{R_{n-1}}^{\alpha}{ }^{Q_{n-1}}\right. \\
& \left.+a S^{Q_{n}}{ }_{Q_{1} R_{n-1}} D_{\alpha, N_{1}}{ }^{M_{1}} D_{Q_{n}}^{\alpha}{ }^{P_{n}}\right]=0,
\end{aligned}
$$

and using the condition of invariance of $S^{P_{n}} Q_{1} R_{n-1}$ this becomes

$$
\Theta^{N_{1}} \Theta^{Q_{1}}\left[\delta_{N_{1}}^{M_{1}} S^{P_{n}}{ }_{Q_{1} R_{n-1}}+a S^{P_{n}} P_{1} R_{n-1} D_{\alpha, N_{1}}{ }^{M_{1}} D_{Q_{1}}^{\alpha}{ }^{P_{1}}\right]=0,
$$

which is eq. (2.20) contracted by $S^{P_{n}} P_{1} R_{n-1}$. We have thus determined all quadratic constraints, and we will show in the following sections that they are all automatically satisfied provided that eq. (2.17) holds and that $a$ is fixed accordingly.

In [26] the trombone deformations were considered together with the deformations resulting from the gauging of a subgroup of the internal symmetry group, and quadratic constraints involving the embedding tensors associated to both deformations were derived in all dimensions from 3 to 6 . If the quadratic constraints allow for the simultaneous presence of both embedding tensors, this would imply the presence of a new class of theories with simultaneously non-vanishing embedding tensors. This was indeed the outcome of the analysis in [26] in any dimension. From our algebraic viewpoint, this corresponds to considering as starting point the more general commutator

$$
\left[R^{\mu, M_{1}}, P_{\nu}\right]=\delta_{\nu}^{\mu}\left(\Theta^{M_{1}} K+a \Theta^{N_{1}} g_{\alpha \beta} D_{N_{1}}^{\alpha M_{1}} R^{\beta}+\Theta_{\alpha}^{M_{1}} R^{\alpha}\right),
$$

where $\Theta_{\alpha}^{M_{1}}$ is the embedding tensor corresponding to the gauging of the internal symmetry. Considering together the analysis of [22] and the one performed in this section, we write more generally the commutator of the 2 -form generator with momentum as

$$
\left[R^{\mu_{1} \mu_{2}, M_{2}}, P_{\nu}\right]=\left(W^{M_{2}} M_{1}+\Theta^{N_{1}} S_{N_{1} M_{1}}^{M_{2}}\right) \delta_{\nu}^{\left[\mu_{1}\right.} R^{\left.\mu_{2}\right], M_{1}},
$$

where $W^{M_{2}} M_{1}$ is related to the embedding tensor $\Theta_{\alpha}^{M_{1}}$ as explained in [22]. One then derives the quadratic constraints of [26] from Jacobi identities. In particular, using eqs. (2.23) and (2.24) the Jacobi identity involving the 2-form and two momentum operators gives

$$
\begin{aligned}
& W^{M_{2}}{ }_{M_{1}} \Theta^{M_{1}}+\Theta^{M_{1}} \Theta^{N_{1}} S^{M_{2}}{ }_{N_{1} M_{1}}=0 \\
& W^{M_{2}}{ }_{M_{1}} \Theta_{\alpha}^{M_{1}}+a W^{M_{2}}{ }_{M_{1}} \Theta^{P_{1}} D_{\alpha, P_{1}}{ }^{M_{1}}+a \Theta^{N_{1}} \Theta^{P_{1}} S^{M_{2}}{ }_{N_{1} M_{1}} D_{\alpha, P_{1}}{ }^{M_{1}} \\
& \quad+\Theta^{N_{1}} \Theta_{\alpha}^{M_{1}} S^{M_{2}}{ }_{N_{1} M_{1}}=0,
\end{aligned}
$$


while the Jacobi identity involving the 1-form generator, momentum and the scalar generator $\Theta^{M_{1}} K+a \Theta^{N_{1}} D_{\alpha, N_{1}}^{M_{1}} R^{\alpha}+\Theta_{\alpha}^{M_{1}} R^{\alpha}$ gives

$$
\begin{aligned}
& \Theta^{N_{1}} \Theta^{Q_{1}}\left[\delta_{N_{1}}^{M_{1}} \delta_{Q_{1}}^{P_{1}}+a D_{\alpha, N_{1}}{ }^{M_{1}} D_{Q_{1}}^{\alpha{ }^{P_{1}}}\right]+\Theta_{\alpha}^{M_{1}} \Theta^{Q_{1}} D_{Q_{1}}^{\alpha{ }^{P_{1}}}=0 \\
& a \Theta^{N_{1}} \Theta_{\alpha}^{P_{1}} D_{\beta, N_{1}}{ }^{M_{1}} f^{\alpha \beta}{ }_{\gamma}-\Theta_{\alpha}^{M_{1}} \Theta_{\beta}^{P_{1}} f^{\alpha \beta}{ }_{\gamma}+a^{2} \Theta^{N_{1}} \Theta^{Q_{1}} D_{\alpha, N_{1}}{ }^{M_{1}}\left(D_{\alpha} D^{\gamma}\right)_{Q_{1}}{ }^{P_{1}} \\
& \quad+a \Theta^{N_{1}} \Theta_{\gamma}^{Q_{1}} D_{\alpha, N_{1}}^{{ }^{M_{1}}} D_{Q_{1}}^{\alpha}{ }^{P_{1}}+a \Theta_{\alpha}^{M_{1}} \Theta^{Q_{1}}\left(D_{\alpha} D^{\gamma}\right)_{Q_{1}}^{{ }^{1}}-\Theta_{\alpha}^{M_{1}} \Theta_{\gamma}^{Q_{1}} D_{Q_{1}}^{\alpha}{ }^{P_{1}}=0
\end{aligned}
$$

One can show that these quadratic constraints exactly reproduce the quadratic constraints in [26] in all cases. In the rest of this paper we will only consider for simplicity of notation the embedding tensor $\Theta^{M_{1}}$ discussed so far in this section. The analysis can be straightforwardly generalised to the case in which both embedding tensors are turned on but it is technically more involved.

The general method to compute the field strengths and gauge transformations of the fields starting from the group element

$$
g=e^{x \cdot P} e^{\Phi_{\mathrm{Og}} K^{\mathrm{Og}}} \ldots . e^{A_{\mu_{1} \ldots \mu_{n}, M_{n}} R^{\mu_{1} \ldots \mu_{n}, M_{n}}} \ldots, e^{A_{\mu, M_{1}} R^{\mu, M_{1}}} e^{\phi_{\alpha} R^{\alpha}} e^{h_{\mu}{ }^{\nu} K^{\mu}{ }_{\nu}}
$$

is analogous to the one of [22]. Using the algebraic relations of eq. (2.5), (2.10) and (2.11) one obtains from the Maurer-Cartan form the field strengths

$$
\begin{aligned}
& F_{\mu_{1} \mu_{2}, M_{1}}=2\left[\partial_{\left[\mu_{1}\right.} A_{\left.\mu_{2}\right], M_{1}}-\frac{1}{2} \Theta^{Q_{1}} A_{\left[\mu_{1}, N_{1}\right.} A_{\left.\mu_{2}\right], P_{1}}\left(\delta_{Q_{1}}^{N_{1}} \delta_{M_{1}}^{P_{1}}+a D_{\alpha, Q_{1}}{ }^{N_{1}} D_{M_{1}}^{\alpha}{ }^{P_{1}}\right)\right. \\
& \left.-\Theta^{N_{1}} S^{M_{2}}{ }_{N_{1} M_{1}} A_{\mu_{1} \mu_{2}, M_{2}}\right] \\
& F_{\mu_{1} \mu_{2} \mu_{3}, M_{2}}=3\left[\partial_{\left[\mu_{1}\right.} A_{\left.\mu_{2} \mu_{3}\right], M_{2}}+\frac{1}{2} \partial_{\left[\mu_{1}\right.} A_{\mu_{2}, M_{1}} A_{\left.\mu_{3}\right], N_{1}} f_{M_{2}}^{M_{1} N_{1}}-A_{\mu_{1} \mu_{2} \mu_{3}, M_{3}} \Theta^{N_{1}} S^{M_{3}}{ }_{N_{1} M_{2}}\right. \\
& -A_{\left[\mu_{1} \mu_{2}, N_{2}\right.} A_{\left.\mu_{3}\right], M_{1}} \Theta^{N_{1}} S^{N_{2}}{ }_{N_{1} P_{1}} f^{P_{1} M_{1}}{ }_{M_{2}} \\
& \left.-\frac{a}{6} A_{\left[\mu_{1}, M_{1}\right.} A_{\mu_{2}, N_{1}} A_{\left.\mu_{3}\right], P_{1}} \Theta^{Q_{1}} D_{\alpha, Q_{1}}{ }^{M_{1}} D_{R_{1}}^{\alpha{ }^{N_{1}}} f^{R_{1} P_{1}}{ }_{M_{2}}\right] \\
& F_{\mu_{1} \mu_{2} \mu_{3} \mu_{4}, M_{3}}=4\left[\partial_{\left[\mu_{1}\right.} A_{\left.\mu_{2} \mu_{3} \mu_{4}\right], M_{3}}-\partial_{\left[\mu_{1}\right.} A_{\mu_{2} \mu_{3}, M_{2}} A_{\left.\mu_{4}\right], M_{1}} f^{M_{1} M_{2}}{ }_{M_{3}}\right. \\
& -\frac{1}{6} \partial_{\left[\mu_{1}\right.} A_{\mu_{2}, M_{1}} A_{\mu_{3}, N_{1}} A_{\left.\mu_{4}\right], P_{1}} f^{M_{1} N_{1}}{ }_{M_{2}} f^{P_{1} M_{2}}{ }_{M_{3}}-A_{\mu_{1} \mu_{2} \mu_{3} \mu_{4}, M_{4}} \Theta^{N_{1}} S^{M_{4}}{ }_{M_{1} M_{3}} \\
& +A_{\left[\mu_{1} \mu_{2} \mu_{3}, N_{3}\right.} A_{\left.\mu_{4}\right], M_{1}} \Theta^{N_{1}} S^{N_{3}}{ }_{N_{1} M_{2}} f^{M_{1} M_{2}}{ }_{M_{3}} \\
& -\frac{1}{2} A_{\left[\mu_{1} \mu_{2}, M_{2}\right.} A_{\left.\mu_{3} \mu_{4}\right], N_{2}} \Theta^{M_{1}} S^{M_{2}}{ }_{M_{1} N_{1}} f^{N_{1} N_{2}}{ }_{M_{3}} \\
& +\frac{1}{2} A_{\left[\mu_{1} \mu_{2}, M_{2}\right.} A_{\mu_{3}, M_{1}} A_{\left.\mu_{4}\right], N_{1}} \Theta^{P_{1}} S^{M_{2}}{ }_{P_{1} Q_{1}} f^{Q_{1} M_{1}}{ }_{N_{2}} f^{N_{1} N_{2}}{ }_{M_{3}} \\
& \left.+\frac{a}{24} A_{\left[\mu_{1}, M_{1}\right.} A_{\mu_{2}, N_{1}} A_{\mu_{3}, P_{1}} A_{\left.\mu_{4}\right], Q_{1}} \Theta^{R_{1}} D_{\alpha, R_{1}}{ }^{M_{1}} D_{S_{1}}^{\alpha{ }^{N_{1}}} f^{S_{1} P_{1}}{ }_{M_{2}} f^{Q_{1} M_{2}}{ }_{M_{3}}\right]
\end{aligned}
$$

for the 1-form, the 2-form and the 3-form potentials. For simplicity we will not consider forms of higher rank in this paper. Acting on the group element of eq. (2.27) we also 
determine the gauge transformations of these fields to be (see Section 2 and Appendix B of [22] for a detailed derivation)

$$
\begin{aligned}
& \delta A_{\mu, M_{1}}=a_{\mu, M_{1}}+\Lambda_{N_{1}} \Theta^{P_{1}}\left(\delta_{P_{1}}^{N_{1}} \delta_{M_{1}}^{Q_{1}}+a D_{\alpha, P_{1}}^{N_{1}} D_{M_{1}}^{\alpha}{ }^{Q_{1}}\right) A_{\mu, Q_{1}} \\
& \delta A_{\mu_{1} \mu_{2}, M_{2}}=a_{\mu_{1} \mu_{2}, M_{2}}+\frac{1}{2} a_{\left[\mu_{1}, M_{1}\right.} A_{\left.\mu_{2}\right], N_{1}} f^{M_{1} N_{1}}{ }_{M_{2}} \\
& \quad+\Lambda_{N_{1}} \Theta^{P_{1}}\left(2 \delta_{P_{1}}^{N_{1}} \delta_{M_{2}}^{Q_{2}}+a D_{\alpha, P_{1}}{ }^{N_{1}} D_{M_{2}}^{\alpha}{ }^{Q_{2}}\right) A_{\mu_{1} \mu_{2}, Q_{2}} \\
& \delta A_{\mu_{1} \mu_{2} \mu_{3}, M_{3}}=a_{\mu_{1} \mu_{2} \mu_{3}, M_{3}}+a_{\left[\mu_{1}, M_{1}\right.} A_{\left.\mu_{2} \mu_{3}\right], M_{2}} f^{M_{1} M_{2}}{ }_{M_{3}} \\
& \quad+\frac{1}{6} a_{\left[\mu_{1}, M_{1}\right.} A_{\mu_{2}, N_{1}} A_{\left.\mu_{3}\right], P_{1}} f^{M_{1} N_{1}}{ }_{N_{2}} f^{P_{1} N_{2}}{ }_{M_{3}} \\
& \quad+\Lambda_{N_{1}} \Theta^{P_{1}}\left(3 \delta_{P_{1}}^{N_{1}} \delta_{M_{3}}^{Q_{3}}+a D_{\alpha, P_{1}}{ }^{N_{1}} D_{M_{3}}^{\alpha}{ }^{Q_{3}}\right) A_{\mu_{1} \mu_{2} \mu_{3}, Q_{3}}
\end{aligned}
$$

where the parameters $a_{\mu_{1} \ldots \mu_{n}, M_{n}}$ are given in terms of the gauge parameters $\Lambda_{\mu_{1} \ldots \mu_{n-1}, M_{n}}$ as

$$
a_{\mu_{1} \ldots \mu_{n}, M_{n}}=\partial_{\left[\mu_{1}\right.} \Lambda_{\left.\mu_{2} \ldots \mu_{n}\right], M_{n}}+\Theta^{M_{1}} S^{M_{n+1}} M_{1} M_{n} \Lambda_{\mu_{1} \ldots \mu_{n}, M_{n+1}} .
$$

Using eqs. (2.17) and (2.20) one can show that the embedding tensor projects $A_{\mu, M_{1}}$ onto the abelian vector transforming as [26]

$$
\delta\left(\Theta^{M_{1}} A_{\mu, M_{1}}\right)=\partial_{\mu}\left(\Theta^{M_{1}} \Lambda_{M_{1}}\right) .
$$

What we are describing is thus an abelian gauging in a formulation which is formally covariant under $E_{11-D}$. All the other vectors are abelian vectors which are also charged with respect to the gauged $U(1)$. Some of these vectors are gauged away by the parameter $\Lambda_{\mu, M_{2}}$, and the hierarchical structure continues to higher rank forms exactly as in the case of the internal gaugings.

We expect the dynamics to arise from duality relations among the field strengths. This was indeed shown in three dimensions in [26] by imposing the closure of the supersymmetry algebra. In $[19,20]$ it was shown that decomposing the $E_{11}$ algebra with respect to $G L(D, \mathbb{R}) \otimes E_{11-D}$ one finds $D-1$ form generators in the same $E_{11-D}$ representation as the embedding tensor $\Theta_{\alpha}^{M_{1}}$ associated to the internal gaugings. Correspondingly, one expects the supersymmetry algebra to close on the corresponding fields, provided that a duality relation between the field strength of the $D-1$ form potential and the embedding tensor holds. This was indeed explicitly shown first in the case of Romans IIA [28, 29], and then in the five dimensional [30] and three dimensional [31] cases. In [32, 31] it was also observed that the $D$ form generators predicted by $E_{11}$ contain in all cases the $E_{11-D}$ representations associated to the quadratic constraints of the embedding tensor $\Theta_{\alpha}^{M_{1}}$. 
The $E_{11}$ spectrum does not possess non-propagating forms (that is forms of rank higher than $D-2)$ in the same representation as the embedding tensor $\Theta^{M_{1}}$, and thus this embedding tensor associated to the trombone deformations discussed in this paper can not be related by duality to an $E_{11}$ form. In [26] it was conjectured that the generators in the representation $G L(D, \mathbb{R})$ of mixed symmetry that we denote by $(1, D-2)$, that is the generators $R^{\mu, \nu_{1} \ldots \nu_{D-2}, M_{1}}$, that are in the same $E_{11-D}$ representation as the 1-form generators and thus as the trombone embedding tensor, might trigger these deformations. As the quadratic constraint of eq. (2.17) projects out the representation $\mathbf{R}_{\mathbf{2}}$ to which the 2-form generators belong, in [26] it was also conjectured that the $E_{11}$ generators $R^{\mu_{1} \mu_{2}, \nu_{1} \ldots \nu_{D-2}, M_{2}}$ in the $(2, D-2)$ representation of $G L(D, \mathbb{R})$ might be associated to these quadratic constraints. Observe that the presence of these generators in the $E_{11}$ spectrum is completely general, as shown in [33]. Indeed these are the first of an infinite chain of so called "dual" $n$-form generators in the $G L(D, \mathbb{R})$ representations $(n, D-2, D-2, \ldots, D-2)$ and in the same $E_{11-D}$ representation $\mathbf{R}_{\mathbf{n}}$ as the $n$-form generators. Their presence is crucial for the universal structure of $E_{11}$ reproducing the gauge algebra of all the form fields in all dimensions. In $[32,31]$ it was observed that in the case of the internal gaugings one can consider a lagrangian formulation in which the $D-1$ forms are Lagrange multipliers for the embedding tensor (so that their field equation implies the constancy of the embedding tensor) and the $D$ forms are Lagrange multipliers for the quadratic constraints. The fact that these forms are present in the gauge algebra is thus intrinsically related to the fact that one expects such a lagrangian formulation to be possible. The theories considered in this paper do not admit a lagrangian formulation, because they result from the gauging of the trombone symmetry, which is not a symmetry of the lagrangian but only of the field equations. Thus we consider the fact that there are no form generators associated to these deformations as completely consistent, and we do not expect any $E_{11}$ generator associated to a non-propagating field to play a role in triggering these deformations.

The main difference with respect to the deformations of the $E_{11, D}^{\text {local }}$ algebra studied in $[22]$ is that in this case the deformation also involves the $G L(D, \mathbb{R})$ generators, and we thus have to consider the gravity sector as well. The modification of the gravity part is precisely as explained in the ten-dimensional gauged IIA case in [27]. Starting from the group element of eq. (2.27) and considering the deformed commutator of eq. (2.5), the $K^{a}{ }_{b}$ term in the Maurer-Cartan form becomes

$$
\left[\left(e^{-1} \partial_{\mu} e\right)_{a b}-\Phi_{\mu \nu}^{\rho} e_{a}^{\nu} e_{\rho b}-\Theta^{M_{1}} A_{\mu, M_{1}} \eta_{a b}\right] K^{a b}
$$


where $\Phi_{\mu \nu}^{\rho}$ is the gravity Og 1 field. Observing that the vierbein transforms under the gauge parameter of the 1 -form $\Lambda_{M_{1}}$ as

$$
e_{\mu}^{a} \rightarrow e^{\Theta^{M_{1}} \Lambda_{M_{1}}} e_{\mu}^{a}
$$

we write the term contracting $K^{a b}$ in eq. (2.32) as

$$
\left(e^{-1} D_{\mu} e\right)_{a b}-\Phi_{\mu \nu}^{\rho} e_{a}^{\nu} e_{\rho b}
$$

where $D_{\mu}$ is the derivative covariantised with respect to the transformation of eq. (2.33), that is $D_{\mu}=\partial_{\mu}-\Theta^{M_{1}} A_{\mu, M_{1}}$. Applying the same arguments of [21] we obtain that imposing that the symmetric part in $a b$ of eq. (2.34) vanishes gives for the antisymmetric part the spin connection

$$
\omega_{\mu}^{a b}=\frac{1}{2} e^{\nu a}\left(D_{\mu} e_{\nu}^{b}-D_{\nu} e_{\mu}{ }^{b}\right)-\frac{1}{2} e^{\nu b}\left(D_{\mu} e_{\nu}{ }^{a}-D_{\nu} e_{\mu}{ }^{a}\right)-\frac{1}{2} e^{\nu a} e^{\rho b}\left(D_{\nu} e_{\rho}^{c}-D_{\rho} e_{\nu}{ }^{c}\right) e_{\mu}{ }^{c},
$$

that is

$$
\tilde{\omega}_{\mu}^{a b}=\omega_{\mu}^{a b}-2 \Theta^{M_{1}} e_{\mu}{ }^{[a} e^{|\nu| b]} A_{\nu, M_{1}} .
$$

If one plugs this into the Maurer-Cartan form and applies the inverse Higgs mechanism at the level of the next gravity Og field, one obtains that the term contracting $K^{a b}{ }_{c}$ is the covariantised Riemann tensor

$$
\tilde{R}_{\mu \nu}^{a b}=2 \partial_{[\mu} \tilde{\omega}_{\nu]}^{a b}+2 \tilde{\omega}_{[\mu}^{a c} \tilde{\omega}_{\nu] c}^{b}
$$

This reproduces exactly the analysis of [26] in the gravity sector.

The rest of this paper will be devoted to a careful analysis of the trombone deformations in each dimensions.

\section{$3 \mathrm{D}=3$}

The massless maximal supergravity theory in three dimensions was constructed in [34]. Its bosonic sector describes 128 scalars parametrising the manifold $E_{8(+8)} / S O(16)$ and the metric. This theory arises from the $E_{11}$ decomposition appropriate to three dimensions, corresponding to the deletion of node 3 in the Dynkin diagram of fig. 1 . We consider the decomposition of the adjoint of $E_{11}$ in terms of $G L(3, \mathbb{R}) \otimes E_{8(8)}$ and we only take into 
account the level zero generators and the positive level generators which are forms of rank at most two, which are

$$
K^{\mu}{ }_{\nu}(\mathbf{1}) \quad R^{\alpha}(\mathbf{2 4 8}) \quad R^{\mu, \alpha}(\mathbf{2 4 8}) \quad R^{\mu_{1} \mu_{2}, M}(\mathbf{3 8 7 5}) \quad R^{\mu_{1} \mu_{2}}(\mathbf{1})
$$

The 1-form generators are in the same $E_{8}$ representation of the scalars, corresponding to the fact that in three dimensions vectors are dual to scalars, while the 2-form generators are in the $\mathbf{1} \oplus \mathbf{3 8 7 5}$.

The commutators of these generators which are relevant for our purpose were given in [22], and are

$$
\begin{aligned}
& {\left[R^{\alpha}, R^{\beta}\right]=f^{\alpha \beta}{ }_{\gamma} R^{\gamma}} \\
& {\left[R^{\alpha}, R^{\mu, \beta}\right]=f^{\alpha \beta}{ }_{\gamma} R^{\mu, \gamma}} \\
& {\left[R^{\alpha}, R^{\mu_{1} \mu_{2}}\right]=0} \\
& {\left[R^{\alpha}, R^{\mu_{1} \mu_{2}, M}\right]=D_{N}^{\alpha M} R^{\mu_{1} \mu_{2}, N}} \\
& {\left[R^{\mu_{1}, \alpha}, R^{\mu_{2}, \beta}\right]=g^{\alpha \beta} R^{\mu_{1} \mu_{2}}+S_{M}^{\alpha \beta} R^{\mu_{1} \mu_{2}, M} .}
\end{aligned}
$$

We take our $E_{8}$ conventions from [35], and thus $g^{\alpha \beta}$ is the Cartan-Killing metric, defined by

$$
g^{\alpha \beta}=-\frac{1}{60} f_{\gamma \delta}^{\alpha} f^{\beta \gamma \delta},
$$

and it is the metric we use to raise $E_{8}$ indices in the adjoint, $D_{N}^{\alpha M}$ are the $E_{8}$ generators in the $\mathbf{3 8 7 5}$ and $S_{M}^{\alpha \beta}$ is an $E_{8}$ invariant tensor symmetric in $\alpha \beta$. The symmetric product of two adjoint representations of $E_{8}$ is

$$
[248 \otimes 248]_{S}=1 \oplus 3875 \oplus \mathbf{2 7 0 0 0}
$$

and we will need the $E_{8}$ projectors

$$
\begin{aligned}
& \mathbb{P}_{\mathbf{1} \alpha \beta}{ }^{\gamma \delta}=\frac{1}{248} g_{\alpha \beta} g^{\gamma \delta} \\
& \mathbb{P}_{\mathbf{3 8 7 5} \alpha \beta}{ }^{\gamma \delta}=\frac{1}{7} \delta_{(\alpha}^{\gamma} \delta_{\beta)}^{\delta}-\frac{1}{14} f^{\sigma \gamma}{ }_{(\alpha} f_{\beta) \sigma}{ }^{\delta}-\frac{1}{56} g_{\alpha \beta} g^{\gamma \delta} \\
& \mathbb{P}_{\mathbf{2 7 0 0 0} \alpha \beta}{ }^{\gamma \delta}=\frac{6}{7} \delta_{(\alpha}^{\gamma} \delta_{\beta)}^{\delta}+\frac{1}{14} f^{\sigma \gamma}{ }_{(\alpha} f_{\beta) \sigma}{ }^{\delta}+\frac{3}{217} g_{\alpha \beta} g^{\gamma \delta},
\end{aligned}
$$

which project the symmetric product of two adjoint indices $\alpha \beta$ into the singlet, the $\mathbf{3 8 7 5}$ and the $\mathbf{2 7 0 0 0}$ respectively. The invariant tensor $S_{M}^{\alpha \beta}$ must satisfy constraints that project 
the symmetric indices $\alpha \beta$ on the $\mathbf{3 8 7 5}$, and from eq. (3.5) one gets [22]

$$
\begin{aligned}
& g_{\alpha \beta} S_{M}^{\alpha \beta}=0 \\
& S_{M}^{\alpha \beta}=-\frac{1}{12} f_{\gamma}^{\epsilon}{ }^{\alpha} f_{\epsilon \delta}{ }^{\beta} S_{M}^{\gamma \delta}
\end{aligned}
$$

Following the general analysis of section 2, we now consider the trombone deformation of this algebra. We start form the deformed commutator

$$
\left[R^{\mu, \alpha}, P_{\nu}\right]=\delta_{\nu}^{\mu}\left(\Theta^{\alpha} K+a f_{\beta \gamma}^{\alpha} \Theta^{\beta} R^{\gamma}\right)
$$

with the parameter $a$ to be determined. We also write the commutators of the 2 -form generators with momentum,

$$
\begin{aligned}
& {\left[R^{\mu_{1} \mu_{2}}, P_{\nu}\right]=b \Theta^{\alpha} \delta_{\nu}^{\left[\mu_{1}\right.} R^{\left.\mu_{2}\right], \beta} g_{\alpha \beta}} \\
& S_{M}^{\gamma \delta}\left[R^{\mu_{1} \mu_{2}, M}, P_{\nu}\right]=c \Theta^{\alpha} \delta_{\nu}^{\left[\mu_{1}\right.} R^{\left.\mu_{2}\right], \beta} \mathbb{P}_{\mathbf{3 8 7 5} \alpha \beta}{ }^{\gamma \delta}
\end{aligned}
$$

with additional parameters $b$ and $c$ to be determined.

We first determine the quadratic constraints resulting from imposing the closure of the Jacobi identities involving the 2-form generator and two momentum operators. This gives the $E_{8}$ case of eq. (2.17), that is

$$
\begin{aligned}
& g_{\alpha \beta} \Theta^{\alpha} \Theta^{\beta}=0 \\
& \mathbb{P}_{\mathbf{3 8 7 5} \alpha \beta}{ }^{\gamma \delta} \Theta^{\alpha} \Theta^{\beta}=0
\end{aligned}
$$

We then impose the closure of the Jacobi identity involving $R^{\mu, \alpha}, R^{\nu, \beta}$ and $P_{\rho}$, obtaining the condition

$$
\left(2-\frac{c}{7}\right) \Theta^{(\alpha} R^{\mu, \beta)}+\left(2 a+\frac{c}{14}\right) \Theta^{\gamma} f_{\gamma \delta}^{(\alpha} f^{|\delta| \beta)} R^{\mu, \epsilon}+\left(\frac{c}{56}-b\right) g^{\alpha \beta} g_{\gamma \delta} \Theta^{\gamma} R^{\mu, \delta}=0
$$

which implies

$$
a=-\frac{1}{2} \quad b=\frac{1}{4} \quad c=14 .
$$

Remarkably, for this value of $a$ the Jacobi identity between the scalar operator $\Theta^{\alpha} K+$ $a f^{\alpha}{ }_{\beta \gamma} \Theta^{\beta} R^{\gamma}$, the 1-form generator $R^{\mu, \alpha}$ and momentum, which is eq. (2.20), becomes

$$
\Theta^{\alpha} \Theta^{\beta}-\frac{1}{2} f_{\gamma \delta}^{\alpha} f_{\epsilon}^{\delta \beta} \Theta^{\gamma} \Theta^{\epsilon}=0
$$

This is exactly the quadratic constraint derived in the field theory analysis of [26]. As it is clear from the projector operators of eq. (3.5), this equation projects the product 
of two embedding tensors on the 27000, and given eq. (3.4), this condition is equivalent to eq. (3.9), which projects out the singlet and the 3875 . This proves in this $E_{8}$ case the statement made in section 2 that the condition resulting from this Jacobi identity is already contained in the "pure spinor constraint" of eq. (2.17).

To summarise, we have obtained the commutators

$$
\begin{aligned}
& {\left[R^{\mu, \alpha}, P_{\nu}\right]=\delta_{\nu}^{\mu}\left(\Theta^{\alpha} K-\frac{1}{2} f^{\alpha}{ }_{\beta \gamma} \Theta^{\beta} R^{\gamma}\right)} \\
& {\left[R^{\mu_{1} \mu_{2}}, P_{\nu}\right]=\frac{1}{4} g_{\alpha \beta} \Theta^{\alpha} \delta_{\nu}^{\left[\mu_{1}\right.} R^{\left.\mu_{2}\right], \beta}} \\
& S_{M}^{\gamma \delta}\left[R^{\mu_{1} \mu_{2}, M}, P_{\nu}\right]=14 \Theta^{\alpha} \delta_{\nu}^{\left[\mu_{1}\right.} R^{\left.\mu_{2}\right], \beta} \mathbb{P}_{\mathbf{3 8 7 5} \alpha \beta}{ }^{\gamma \delta},
\end{aligned}
$$

and we normalise the invariant tensor $S_{M}^{\alpha \beta}$ in such a way that

$$
S_{M}^{\alpha \beta} S_{\alpha \beta, N}=\delta_{M N}
$$

so that the last of eqs. (3.13) can be written

$$
\left[R^{\mu_{1} \mu_{2}, M}, P_{\nu}\right]=14 \Theta^{\alpha} \delta_{\nu}^{\left[\mu_{1}\right.} R^{\left.\mu_{2}\right], \beta} S_{\alpha \beta}^{M}
$$

To conclude this section, we apply the general formulae of section 2 to derive the field strengths and gauge transformations of the fields. We consider the group element

$$
g=e^{x \cdot P} e^{A_{\mu_{1} \mu_{2}} R^{\mu_{1} \mu_{2}}} e^{A_{\mu_{1} \mu_{2}, M} R^{\mu_{1} \mu_{2}, M}} e^{A_{\mu, \alpha} R^{\mu, \alpha}} e^{\phi_{\alpha} R^{\alpha}} e^{h_{\mu}{ }^{\nu} K_{\nu}^{\mu}}
$$

and we compute the Maurer-Cartan form and the gauge transformations using the commutators of eq. (3.2) and (3.13). This gives the field strength for the 1-form

$$
\begin{aligned}
& F_{\mu_{1} \mu_{2}, \alpha}=2\left[\partial_{\left[\mu_{1}\right.} A_{\left.\mu_{2}\right], \alpha}-\frac{1}{2} \Theta^{\beta} A_{\left[\mu_{1}, \beta\right.} A_{\left.\mu_{2}\right], \alpha}+\frac{1}{4} \Theta^{\gamma} f^{\beta}{ }_{\gamma \delta} f^{\delta \epsilon}{ }_{\alpha} A_{\left[\mu_{1}, \beta\right.} A_{\left.\mu_{2}\right], \epsilon}\right. \\
& \left.-\frac{1}{4} \Theta_{\alpha} A_{\mu_{1} \mu_{2}}-14 \Theta^{\beta} S_{\alpha \beta}^{M} A_{\mu_{1} \mu_{2}, M}\right],
\end{aligned}
$$

transforming covariantly under the gauge transformations

$$
\begin{aligned}
& \delta A_{\mu, \alpha}=a_{\mu, \alpha}+\Lambda_{\beta} \Theta^{\beta} A_{\mu, \alpha}-\frac{1}{2} \Lambda_{\delta} \Theta^{\beta} f_{\beta \gamma}^{\delta} f_{\alpha}^{\gamma \sigma} A_{\mu, \sigma} \\
& \delta A_{\mu_{1} \mu_{2}}=\partial_{\left[\mu_{1}\right.} \Lambda_{\left.\mu_{2}\right]}+\frac{1}{2} g^{\alpha \beta} a_{\left[\mu_{1}, \alpha\right.} A_{\left.\mu_{2}\right], \beta}+2 \Lambda_{\alpha} \Theta^{\alpha} A_{\mu_{1} \mu_{2}} \\
& \delta A_{\mu_{1} \mu_{2}, M}=\partial_{\left[\mu_{1}\right.} \Lambda_{\left.\mu_{2}\right], M}+\frac{1}{2} S_{M}^{\alpha \beta} a_{\left[\mu_{1}, \alpha\right.} A_{\left.\mu_{2}\right], \beta}+2 \Lambda_{\alpha} \Theta^{\alpha} A_{\mu_{1} \mu_{2}, M} \\
& -\frac{1}{2} \Lambda_{\alpha} \Theta^{\beta} f^{\alpha}{ }_{\beta \gamma} D_{M}^{\gamma}{ }^{N} A_{\mu_{1} \mu_{2}, N}
\end{aligned}
$$


where $D_{M}^{\alpha}{ }^{N}$ are the $E_{8}$ generators in the 3875 and

$$
a_{\mu, \alpha}=\partial_{\mu} \Lambda_{\alpha}+\frac{1}{4} \Theta_{\alpha} \Lambda_{\mu}+14 \Theta^{\beta} S_{\alpha \beta}^{M} \Lambda_{\mu, M} .
$$

We can also compute the field strengths of the 2-form potentials up to terms involving the 3 -form potential. The result is

$$
\begin{aligned}
& F_{\mu_{1} \mu_{2} \mu_{3}}=3\left[\partial_{\left[\mu_{1}\right.} A_{\left.\mu_{2} \mu_{3}\right]}+\frac{1}{2} g^{\alpha \beta} \partial_{\left[\mu_{1}\right.} A_{\mu_{2}, \alpha} A_{\left.\mu_{3}\right], \beta}-\frac{1}{4} \Theta^{\alpha} A_{\left[\mu_{1} \mu_{2}\right.} A_{\left.\mu_{3}\right], \alpha}\right. \\
& \left.-14 \Theta^{\alpha} S_{\alpha \beta}^{M} A_{\left[\mu_{1} \mu_{2}, M\right.} A_{\left.\mu_{3}\right]}^{\beta}+\frac{1}{12} A_{\left[\mu_{1}, \alpha\right.} A_{\mu_{2}, \beta} A_{\left.\mu_{3}\right], \gamma} \Theta^{\delta} f^{\alpha}{ }_{\delta \sigma} f^{\sigma \beta \gamma}\right] \\
& F_{\mu_{1} \mu_{2} \mu_{3}, M}=3\left[\partial_{\left[\mu_{1}\right.} A_{\left.\mu_{2} \mu_{3}\right], M}+\frac{1}{2} S_{M}^{\alpha \beta} \partial_{\left[\mu_{1}\right.} A_{\mu_{2}, \alpha} A_{\left.\mu_{3}\right], \beta}-\frac{1}{4} A_{\left[\mu_{1} \mu_{2}\right.} A_{\left.\mu_{3}\right], \alpha} \Theta_{\beta} S_{M}^{\alpha \beta}\right. \\
& \left.\quad-14 A_{\left[\mu_{1} \mu_{2}, N\right.} A_{\left.\mu_{3}\right], \alpha} \Theta^{\beta} S_{\beta \gamma}^{N} S_{M}^{\alpha \gamma}+\frac{1}{12} A_{\left[\mu_{1}, \alpha\right.} A_{\mu_{2}, \beta} A_{\left.\mu_{3}\right], \gamma} \Theta^{\delta} f_{\delta \sigma}^{\alpha} f^{\sigma \beta}{ }_{\epsilon} S_{M}^{\epsilon \gamma}\right]
\end{aligned}
$$

To prove the gauge covariance of these field strengths of the 2 -forms one must include the 3 -forms and determine their gauge transformations.

\section{$4 \quad \mathrm{D}=4$}

The deletion of node 4 in the $E_{11}$ Dynkin diagram of fig. 1 results in a decomposition of $E_{11}$ with respect to $G L(4, \mathbb{R}) \otimes E_{7(7)}$, which is relevant for the four-dimensional theory. The massless maximal supergravity theory was constructed in [3], and possesses an $E_{7(7)}$ on-shell symmetry (recently, it was shown that this theory also admits a lagrangian formulation in which the $E_{7(7)}$ symmetry is manifest [36]). The general gaugings of the internal symmetry $E_{7(7)}$ of this theory were constructed in [11] using the embedding tensor formalism, and in [22] it was shown that all these gaugings correspond to deformations of the four-dimensional local $E_{11}$ algebra.

We use the notation of [22], which we now review. We consider the level zero generators and the form generators, which are

$$
\begin{aligned}
& K_{\nu}^{\mu}(\mathbf{1}) \quad R^{\alpha}(\mathbf{1 3 3}) \quad R^{\mu, M}(\mathbf{5 6}) R^{\mu_{1} \mu_{2}, \alpha}(\mathbf{1 3 3}) \\
& R^{\mu_{1} \mu_{2} \mu_{3}, A}(\mathbf{9 1 2}) \quad R^{\mu_{1} \ldots \mu_{4}, \alpha \beta}(\mathbf{8 6 4 5} \oplus \mathbf{1 3 3})
\end{aligned}
$$

where the numbers in brackets denote the corresponding $E_{7}$ representation. The indices $M$, denoting the $\mathbf{5 6}$, are raised and lowered by the antisymmetric invariant metric $\Omega^{M N}$ according to

$$
V^{M}=\Omega^{M N} V_{N} \quad V_{M}=V^{N} \Omega_{N M}
$$


which implies

$$
\Omega^{M N} \Omega_{N P}=-\delta_{P}^{M}
$$

The generator

$$
D^{\alpha, M N}=\Omega^{M P} D_{P}^{\alpha N}
$$

is symmetric in $M N$.

The commutators between the scalars and the other generators are dictated by the $E_{7}$ representation that the generators carry. The other $E_{11}$ commutation relations which will be relevant in this paper are [22]

$$
\begin{aligned}
& {\left[R^{\mu_{1}, M}, R^{\mu_{2}, N}\right]=D_{\alpha}^{M N} R^{\mu_{1} \mu_{2}, \alpha}} \\
& {\left[R^{\mu_{1}, M}, R^{\mu_{2} \mu_{3}, \alpha}\right]=S_{A}^{M \alpha} R^{\mu_{1} \mu_{2} \mu_{3}, A}} \\
& {\left[R^{\mu_{1} \mu_{2}, \alpha}, R^{\mu_{3} \mu_{4}, \beta}\right]=R^{\mu_{1} \ldots \mu_{4}, \alpha \beta}} \\
& {\left[R^{\mu_{1}, M}, R^{\mu_{2} \mu_{3} \mu_{4}, A}\right]=C_{\alpha \beta}^{M A} R^{\mu_{1} \ldots \mu_{4}, \alpha \beta},}
\end{aligned}
$$

and the Jacobi identities as well as the condition of invariance under $E_{7}$ imply that $S_{A}^{M \alpha}$ satisfies $[22]$

$$
D_{\alpha}^{(M N} S_{A}^{P) \alpha}=0
$$

and

$$
D_{\alpha, M}^{N} S_{A}^{M \alpha}=0
$$

These conditions project the $M \alpha$ indices of $S_{A}^{M \alpha}$ along the $\mathbf{9 1 2}$, as can be seen applying the projector operator

$$
\mathbb{P}_{\mathbf{9 1 2} \alpha N}^{M \beta}=\frac{4}{7} D_{N}^{\beta P} D_{\alpha P}{ }^{M}-\frac{12}{7} D_{\alpha N}{ }^{P} D_{P}^{\beta M}+\frac{1}{7} \delta_{N}^{M} \delta_{\alpha}^{\beta}
$$

Following [7], we are using the metric

$$
g^{\alpha \beta}=D_{M}^{\alpha N} D_{N}^{\beta M}
$$

to raise and lower indices in the adjoint. This metric is proportional to the Cartan-Killing metric, as can be seen from

$$
f_{\alpha \beta \gamma} f^{\alpha \beta \delta}=-3 \delta_{\gamma}^{\delta}
$$

The other invariant tensor $C_{\alpha \beta}^{M A}$ satisfies

$$
S_{A}^{M \alpha} C_{\beta \gamma}^{N A}+S_{A}^{N \alpha} C_{\beta \gamma}^{M A}+\delta_{[\beta}^{\alpha} D_{\gamma]}^{M N}=0 .
$$


Including also the momentum operator and the Og operators one constructs the algebra $E_{11,4}^{\text {local }}$ from which one derives the field strengths and gauge transformations of all the fields of the massless maximal supergravity theory in four dimensions [22].

We now consider the trombone deformation of the $E_{11,4}^{\text {local }}$ algebra. We consider as our starting point the commutator

$$
\left[R^{\mu, M}, P_{\nu}\right]=\delta_{\nu}^{\mu}\left(\Theta^{M} K+a \Theta^{N} D_{\alpha, N}{ }^{M} R^{\alpha}\right)
$$

The Jacobi identity among $R^{\mu, M}, R^{\nu, N}$ and $P_{\rho}$ implies that

$$
\left[R^{\mu_{1} \mu_{2}, \alpha}, P_{\nu}\right]=-16 \Theta^{M} D_{M N}^{\alpha} \delta_{\nu}^{\left[\mu_{1}\right.} R^{\left.\mu_{2}\right], N},
$$

and also fixes the parameter $a$ in eq. (4.12) to be

$$
a=-8 .
$$

The Jacobi identity among $R^{\mu_{1}, M}, R^{\mu_{2} \mu_{3}, \alpha}$ and $P_{\nu}$ then gives

$$
S_{A}^{M \alpha}\left[R^{\mu_{1} \mu_{2} \mu_{3}, A}, P_{\nu}\right]=14 \mathbb{P}_{\mathbf{9 1 2} \gamma N}^{M \delta} g^{\alpha \gamma} g_{\beta \delta} \Theta^{N} \delta_{\nu}^{\left[\mu_{1}\right.} R^{\left.\mu_{2} \mu_{3}\right], \beta} .
$$

In deriving these results we have made use of the $E_{7}$ identity

$$
D_{\beta M}{ }^{N} D_{P}^{\beta Q}=\frac{1}{12} \delta_{M}^{Q} \delta_{P}^{N}+\frac{1}{24} \delta_{M}^{N} \delta_{P}^{Q}-\frac{1}{24} \Omega^{N Q} \Omega_{M P}+D_{\beta}^{N Q} D_{M P}^{\beta} .
$$

The $E_{11}$-based derivation of the $E_{7}$ relations of eqs. (4.8) and (4.16) can be found in Appendix A of ref. [22]. The Jacobi identity involving two 2-form generators and momentum gives

$$
\left[R^{\mu_{1} \ldots \mu_{4}, \alpha \beta}, P_{\nu}\right]=-32 \Theta^{M} D_{M N}^{[\alpha} S_{A}^{\beta] N} \delta_{\nu}^{\left[\mu_{1}\right.} R^{\left.\mu_{2} \mu_{3} \mu_{4}\right], A},
$$

and one can show that the Jacobi identity involving the 1-form, the 3-form and momentum is also automatically satisfied.

We now consider the quadratic constraints. The Jacobi identity involving $R^{\mu_{1} \mu_{2}, \alpha}$ and two momentum operators gives the $E_{7}$ case of the "pure spinor condition" of eq. (2.17), which is

$$
\Theta^{M} \Theta^{N} D_{M N}^{\alpha}=0,
$$

which again coincides with the quadratic constraint of [26]. Using the $E_{7}$ relations of Appendix A of ref. [22], some of which we have reviewed in this paper, one can show that 
this constraint automatically implies that the Jacobi identity involving $R^{\mu_{1} \mu_{2} \mu_{3}, A}$ and two momentum operators is automatically satisfied.

Finally, we consider the Jacobi identity among the scalar generator $\Theta^{M} K+a \Theta^{N} D_{\alpha, N}{ }^{M} R^{\alpha}$, the 1 -form $R^{\mu, M}$ and $P_{\nu}$. A simple computation shows that for the value $a=-8$ as in eq. (4.14) already determined imposing the closure of other Jacobi identities, this Jacobi identity is automatically satisfied if the "pure spinor condition" of eq. (4.18) holds.

To summarise, we have obtained the commutators

$$
\begin{aligned}
& {\left[R^{\mu, M}, P_{\nu}\right]=\delta_{\nu}^{\mu}\left(\Theta^{M} K-8 \Theta^{N} D_{\alpha, N}^{M} R^{\alpha}\right)} \\
& {\left[R^{\mu_{1} \mu_{2}, \alpha}, P_{\nu}\right]=-16 \Theta^{M} D_{M N}^{\alpha} \delta_{\nu}^{\left[\mu_{1}\right.} R^{\left.\mu_{2}\right], N}} \\
& S_{A}^{M \alpha}\left[R^{\mu_{1} \mu_{2} \mu_{3}, A}, P_{\nu}\right]=14 \mathbb{P}_{\mathbf{9 1 2} \gamma N}^{M \delta} g^{\alpha \gamma} g_{\beta \delta} \Theta^{N} \delta_{\nu}^{\left[\mu_{1}\right.} R^{\left.\mu_{2} \mu_{3}\right], \beta} \\
& {\left[R^{\mu_{1} \ldots \mu_{4}, \alpha \beta}, P_{\nu}\right]=-32 \Theta^{M} D_{M N}^{[\alpha} S_{A}^{\beta] N} \delta_{\nu}^{\left[\mu_{1}\right.} R^{\left.\mu_{2} \mu_{3} \mu_{4}\right], A}}
\end{aligned} .
$$

Normalising the invariant tensor $S_{A}^{M \alpha}$ in such a way that

$$
S_{A}^{M \alpha} S_{M \alpha}^{B}=\delta_{A}^{B}
$$

the third equation in (4.19) can be rewritten as

$$
\left[R^{\mu_{1} \mu_{2} \mu_{3}, A}, P_{\nu}\right]=14 \Theta^{M} S_{M \alpha}^{A} \delta_{\nu}^{\left[\mu_{1}\right.} R^{\left.\mu_{2} \mu_{3}\right], \alpha} .
$$

We now consider the group element

$$
g=e^{x \cdot P} e^{A_{\mu_{1} \ldots \mu_{4}, \alpha \beta} R^{\mu_{1} \ldots \mu_{4}, \alpha \beta}} \ldots e^{A_{\mu, M} R^{\mu, M}} e^{\phi_{\alpha} R^{\alpha}} e^{h_{\mu}{ }^{\nu} K^{\mu}{ }_{\nu}},
$$

and applying the general formulae of section 2 to derive the field strengths and gauge transformations of the fields, we get

$$
\begin{aligned}
& F_{\mu_{1} \mu_{2}, M}=2\left[\partial_{\left[\mu_{1}\right.} A_{\left.\mu_{2}\right], M}+\frac{1}{6} \Theta^{N} A_{\left[\mu_{1}, M\right.} A_{\left.\mu_{2}\right], N}-\frac{1}{6} \Theta_{M} \Omega^{N P} A_{\left[\mu_{1}, N\right.} A_{\left.\mu_{2}\right], P}\right. \\
& \left.\quad+16 \Theta^{N} D_{M N}^{\alpha} A_{\mu_{1} \mu_{2}, \alpha}\right] \\
& F_{\mu_{1} \mu_{2} \mu_{3}, \alpha}=3\left[\partial_{\left[\mu_{1}\right.} A_{\left.\mu_{2} \mu_{3}\right], \alpha}+\frac{1}{2} \partial_{\left[\mu_{1}\right.} A_{\mu_{2}, M} A_{\left.\mu_{3}\right], N} D_{\alpha}^{M N}-14 \Theta^{M} S_{M \alpha}^{A} A_{\mu_{1} \mu_{2} \mu_{3}, A}\right. \\
& \left.\quad+16 A_{\left[\mu_{1} \mu_{2}, \beta\right.} A_{\left.\mu_{3}\right], M} \Theta^{N} D_{N P}^{\beta} D_{\alpha}^{M P}+\frac{4}{3} A_{\left[\mu_{1}, M\right.} A_{\mu_{2}, N} A_{\left.\mu_{3}\right], P} \Theta^{Q} D_{\beta, Q}{ }^{M} D_{R}^{\beta N} D_{\alpha}^{R P}\right] \\
& F_{\mu_{1} \mu_{2} \mu_{3} \mu_{4}, A}=4\left[\partial_{\left[\mu_{1}\right.} A_{\left.\mu_{2} \mu_{3} \mu_{4}\right], A}-\partial_{\left[\mu_{1}\right.} A_{\mu_{2} \mu_{3}, \alpha} A_{\left.\mu_{4}\right], M} S_{A}^{M \alpha}\right. \\
& \quad-\frac{1}{6} \partial_{\left[\mu_{1}\right.} A_{\mu_{2}, M} A_{\mu_{3}, N} A_{\left.\mu_{4}\right], P} D_{\alpha}^{M N} S_{A}^{P \alpha}+32 \Theta^{M} D_{M N}^{[\alpha} S_{A}^{\beta] N} A_{\mu_{1} \ldots \mu_{4}, \alpha \beta} \\
& \quad+14 A_{\left[\mu_{1} \mu_{2} \mu_{3}, B\right.} A_{\left.\mu_{4}\right], M} \Theta^{N} S_{N \alpha}^{B} S_{A}^{M \alpha}+8 A_{\left[\mu_{1} \mu_{2}, \alpha\right.} A_{\left.\mu_{3} \mu_{4}\right], \beta} \Theta^{M} D_{M N}^{\alpha} S_{A}^{N \beta} \\
& \quad-8 A_{\left[\mu_{1} \mu_{2}, \alpha\right.} A_{\mu_{3}, M} A_{\left.\mu_{4}\right], N} \Theta^{P} D_{P Q}^{\alpha} D_{\beta}^{Q M} S_{A}^{N \beta} \\
& \left.\quad-\frac{1}{3} A_{\left[\mu_{1}, M\right.} A_{\mu_{2}, N} A_{\mu_{3}, P} A_{\left.\mu_{4}\right], Q} \Theta^{R} D_{\alpha, R}^{M} D_{S}^{\alpha N} D_{\beta}^{S P} S_{A}^{Q \beta}\right]
\end{aligned}
$$


These field strengths transform covariantly under the gauge transformations

$$
\begin{aligned}
& \delta A_{\mu, M}=a_{\mu, M}+\Lambda_{N} \Theta^{N} A_{\mu, M}-b \Lambda_{N} \Theta^{P} D_{\alpha, P}{ }^{N} D_{M}^{\alpha} A_{\mu, Q} \\
& \delta A_{\mu_{1} \mu_{2}, \alpha}=a_{\mu_{1} \mu_{2}, \alpha}+\frac{1}{2} a_{\left[\mu_{1}, M\right.} A_{\left.\mu_{2}\right], N} D_{\alpha}^{M N}+2 \Lambda_{M} \Theta^{M} A_{\mu_{1} \mu_{2}, \alpha}-8 \Lambda_{N} \Theta^{P} D_{\beta, P}{ }^{N} f^{\beta \gamma}{ }_{\alpha} A_{\mu_{1} \mu_{2}, \gamma} \\
& \delta A_{\mu_{1} \mu_{2} \mu_{3}, A}=\partial_{\left[\mu_{1}\right.} \Lambda_{\left.\mu_{2} \mu_{3}\right], A}+a_{\left[\mu_{1}, M\right.} A_{\left.\mu_{2} \mu_{3}\right], \alpha} S_{A}^{M \alpha}+\frac{1}{6} a_{\left[\mu_{1}, M\right.} A_{\mu_{2}, N} A_{\left.\mu_{3}\right], P} D_{\alpha}^{M N} S_{A}^{P \alpha} \\
& \quad+3 \Lambda_{M} \Theta^{M} A_{\mu_{1} \mu_{2} \mu_{3}, A}-8 \Lambda_{N} \Theta^{P} D_{\alpha, P}{ }^{N} D_{A}^{\alpha B} A_{\mu_{1} \mu_{2} \mu_{3}, B}
\end{aligned}
$$

where the parameters $a_{\mu_{1} \ldots \mu_{n}, M_{n}}$ are as in eq. (2.30), which gives

$$
\begin{aligned}
& a_{\mu, M}=\partial_{\mu} \Lambda_{M}-16 \Theta^{N} D_{M N}^{\alpha} \Lambda_{\mu, \alpha} \\
& a_{\mu_{1} \mu_{2}, \alpha}=\partial_{\left[\mu_{1}\right.} \Lambda_{\left.\mu_{2}\right], \alpha}+14 \Theta^{M} S_{M \alpha}^{A} \Lambda_{\mu_{1} \mu_{2}, A} .
\end{aligned}
$$

This concludes our four-dimensional analysis.

\section{$5 \quad \mathrm{D}=5$}

The maximal massless supergravity theory in five dimensions was derived in [4]. Its bosonic sector describes 42 scalars parametrising the manifold $E_{6(+6)} / U S p(8)$, the metric and a 1form in the 27. This is described by the decomposition of $E_{11}$ corresponding to the deletion of node 5 in fig. 1. The level zero generators and the form generators up to the 4 -form included that occur in this decomposition of $E_{11}$ with respect to $G L(5, \mathbb{R}) \otimes E_{6}$ are $[19,21]$

$$
K_{\nu}^{\mu}(\mathbf{1}) R^{\alpha}(\mathbf{7 8}) \quad R^{\mu, M}(\overline{\mathbf{2 7}}) \quad R^{\mu_{1} \mu_{2}}{ }_{M}(\mathbf{2 7}) R^{\mu_{1} \mu_{2} \mu_{3}, \alpha}(\mathbf{7 8}) R^{\mu_{1} \mu_{2} \mu_{3} \mu_{4}}{ }_{M N}(\overline{\mathbf{3 5 1}})
$$

where we devote in brackets the $E_{6}$ representation of each generator.

We use the notations of [22], which we now partly review. The commutators between the scalars and the other generators are dictated by the $E_{6}$ representation that the generators carry. The other $E_{11}$ commutation relations which will be relevant in this paper are [22]

$$
\begin{aligned}
& {\left[R^{\mu_{1}, M}, R^{\mu_{2}, N}\right]=d^{M N P} R^{\mu_{1} \mu_{2}}{ }_{P}} \\
& {\left[R^{\mu_{1}, N}, R^{\mu_{2} \mu_{3}}{ }_{M}\right]=g_{\alpha \beta}\left(D^{\alpha}\right)_{M}{ }^{N} R^{\mu_{1} \mu_{2} \mu_{3}, \beta}} \\
& {\left[R^{\mu_{1} \mu_{2}}{ }_{M}, R^{\mu_{3} \mu_{4}}{ }_{N}\right]=R^{\mu_{1} \mu_{2} \mu_{3} \mu_{4}}{ }_{M N}} \\
& {\left[R^{\mu_{1}, P}, R^{\mu_{2} \mu_{3} \mu_{4}, \alpha}\right]=S^{\alpha P, M N} R^{\mu_{1} \mu_{2} \mu_{3} \mu_{4}}{ }_{M N}}
\end{aligned}
$$

where $d^{M N P}$ is the completely symmetric invariant tensor of $E_{6}$ and $g^{\alpha \beta}$ is the metric that we use to raise and lower indices in the adjoint defined by the relation

$$
D_{M}^{\alpha N} D_{N}^{\beta M}=g^{\alpha \beta}
$$


which is proportional to the Cartan-Killing metric as it is evident from

$$
f_{\alpha \beta \gamma} f^{\alpha \beta \delta}=-4 \delta_{\gamma}^{\delta}
$$

where $f^{\alpha \beta \gamma}$ are the structure constants of $E_{6}$. The other $E_{6}$ invariant tensor $S^{\alpha P, M N}$ that occurs in eq. (5.2) is defined by

$$
S^{\alpha M, N P}=-3 D_{Q}^{\alpha[N} d^{P] M Q}
$$

In the following we will also need the conjugate of this invariant tensor, that is

$$
S_{M, N P}^{\alpha}=-3 D_{[N}^{\alpha}{ }^{Q} d_{P] M Q}
$$

We now consider the trombone deformations of this algebra. As explained in section 2, we consider as our starting point the commutation relation

$$
\left[R^{\mu, M}, P_{\nu}\right]=\delta_{\nu}^{\mu}\left(\Theta^{M} K+a \Theta^{N} D_{\alpha, N}{ }^{M} R^{\alpha}\right)
$$

with the parameter $a$ to be determined. We proceed as in the previous cases, imposing the closure of the Jacobi identities involving two form generators and momentum. This determines the parameter $a$ to be

$$
a=-\frac{9}{2},
$$

and it also determines all the commutators of the other form generators with momentum. Summarising, we get the commutators

$$
\begin{aligned}
& {\left[R^{\mu, M}, P_{\nu}\right]=\delta_{\nu}^{\mu}\left(\Theta^{M} K-\frac{9}{2} \Theta^{N} D_{\alpha, N}{ }^{M} R^{\alpha}\right)} \\
& {\left[R^{\mu_{1} \mu_{2}}{ }_{M}, P_{\nu}\right]=15 d_{M N P} \Theta^{N} \delta_{\nu}^{\left[\mu_{1}\right.} R^{\left.\mu_{2}\right], P}} \\
& {\left[R^{\mu_{1} \mu_{2} \mu_{3}, \alpha}, P_{\nu}\right]=\frac{27}{2} \Theta^{M} D_{M}^{\alpha}{ }^{N} \delta_{\nu}^{\left[\mu_{1}\right.} R^{\left.\mu_{2} \mu_{3}\right]}{ }_{N}} \\
& {\left[R^{\mu_{1} \mu_{2} \mu_{3} \mu_{4}}{ }_{M N}, P_{\nu}\right]=10 \Theta^{P} S_{P, M N}^{\alpha} \delta_{\nu}^{\left[\mu_{1}\right.} R_{\alpha}^{\left.\mu_{2} \mu_{3} \mu_{4}\right]},}
\end{aligned}
$$

where the invariant tensor $S_{P, M N}^{\alpha}$ is defined in eq. (5.6).

We now consider the quadratic constraints. The constraint of eq. (2.17) becomes in this $E_{6}$ case

$$
\Theta^{M} \Theta^{N} d_{M N P}=0
$$

which is precisely the $E_{6}$ "pure spinor constraint" obtained in [26]. All the other Jacobi identities give constraints that are all contained in this condition. In particular, the Jacobi 
identity involving the scalar generator $\Theta^{M} K-\frac{9}{2} \Theta^{N} D_{\alpha, N}{ }^{M} R^{\alpha}$, the 1-form generator and momentum is implied by eq. (5.10) using the $E_{6}$ relation

$$
g_{\alpha \beta} D_{M}^{\alpha}{ }^{N} D_{P}^{\beta Q}=\frac{1}{6} \delta_{P}^{N} \delta_{M}^{Q}+\frac{1}{18} \delta_{M}^{N} \delta_{P}^{Q}-\frac{5}{3} d^{N Q R} d_{M P R} .
$$

whose derivation based on $E_{11}$ is presented in Appendix A of ref. [22].

We now determine the field strengths and gauge transformations of the fields as they result from the deformed local $E_{11}$ group element. We start from the group element

$$
g=e^{x \cdot P} e^{A_{\mu_{1} \ldots \mu_{4}}^{M N} R_{M N}^{\mu_{1} \ldots \mu_{4}}} e^{A_{\mu_{1} \mu_{2} \mu_{3}, \alpha} R^{\mu_{1} \mu_{2} \mu_{3}, \alpha}} e^{A_{\mu_{1} \mu_{2}}^{M} R_{M}^{\mu_{1} \mu_{2}}} e^{A_{\mu, M} R^{\mu, M}} e^{\phi_{\alpha} R^{\alpha}} e^{h_{\mu}{ }^{\nu} K^{\mu}{ }_{\nu}}
$$

and using the general formulae of section 2 as well as the results in this section we find

$$
\begin{aligned}
& F_{\mu_{1} \mu_{2}, M}=2\left[\partial_{\left[\mu_{1}\right.} A_{\left.\mu_{2}\right], M}-\frac{3}{4} \Theta^{N} A_{\left[\mu_{1}, N\right.} A_{\left.\mu_{2}\right], M}-15 \Theta^{N} d_{M N P} A_{\mu_{1} \mu_{2}}^{P}\right] \\
& F_{\mu_{1} \mu_{2} \mu_{3}}^{M}=3\left[\partial_{\left[\mu_{1}\right.} A_{\left.\mu_{2} \mu_{3}\right]}^{M}+\frac{1}{2} \partial_{\left[\mu_{1}\right.} A_{\mu_{2}, N} A_{\left.\mu_{3}\right], P} d^{M N P}-\frac{27}{2} \Theta^{N} D_{N}^{\alpha M} A_{\mu_{1} \mu_{2} \mu_{3}, \alpha}\right. \\
& \left.\quad-15 A_{\left[\mu_{1} \mu_{2}\right.}^{N} A_{\left.\mu_{3}\right], P} \Theta^{Q} d_{Q N R} d^{R P M}+\frac{3}{4} A_{\left[\mu_{1}, N\right.} A_{\mu_{2}, P} A_{\left.\mu_{3}\right], Q} \Theta^{R} D_{\alpha, R}{ }^{N} D_{S}^{\alpha P} d^{S Q M}\right] \\
& F_{\mu_{1} \mu_{2} \mu_{3} \mu_{4}}^{\alpha}=4\left[\partial_{\left[\mu_{1}\right.} A_{\left.\mu_{2} \mu_{3} \mu_{4}\right]}^{\alpha}-\partial_{\left[\mu_{1}\right.} A_{\mu_{2} \mu_{3}}^{M} A_{\left.\mu_{4}\right], N} D_{M}^{\alpha}-\frac{1}{6} \partial_{\left[\mu_{1}\right.} A_{\mu_{2}, M} A_{\mu_{3}, N} A_{\left.\mu_{4}\right], P} d^{M N Q} D_{Q}^{\alpha P}\right. \\
& -10 \Theta^{P} S_{P, M N}^{\alpha} A_{\mu_{1} \ldots \mu_{4}}^{M N}+\frac{27}{2} A_{\left[\mu_{1} \mu_{2} \mu_{3}, \beta\right.} A_{\left.\mu_{4}\right], M} \Theta^{N} D_{N}^{\beta P} D_{P}^{\alpha M} \\
& \quad-\frac{15}{2} A_{\left[\mu_{1} \mu_{2}\right.}^{M} A_{\left.\mu_{3} \mu_{4}\right]}^{N} \Theta^{P} d_{P M Q} D_{N}^{\alpha Q}+\frac{15}{2} A_{\left[\mu_{1} \mu_{2}\right.}^{M} A_{\mu_{3}, N} A_{\left.\mu_{4}\right], P} \Theta^{Q} d_{Q M R} d^{R N S} D_{S}^{\alpha P} \\
& \left.-\frac{3}{16} A_{\left[\mu_{1}, M\right.} A_{\mu_{2}, N} A_{\mu_{3}, P} A_{\left.\mu_{4}\right], Q} \Theta^{R} D_{\beta, R}^{M} D_{S}^{\beta N} d^{S P T} D_{T}^{\alpha Q}\right]
\end{aligned}
$$

These field strengths are covariant with respect to the gauge transformations

$$
\begin{aligned}
& \delta A_{\mu, M}=a_{\mu, M}+\Lambda_{N} \Theta^{N} A_{\mu, M}-\frac{9}{2} \Lambda_{N} \Theta^{P} D_{\alpha, P}{ }^{N} D_{M}^{\alpha} A_{\mu, Q} \\
& \delta A_{\mu_{1} \mu_{2}}^{M}=a_{\mu_{1} \mu_{2}}^{M}+\frac{1}{2} a_{\left[\mu_{1}, N\right.} A_{\left.\mu_{2}\right], P} d^{M N P}+2 \Lambda_{N} \Theta^{N} A_{\mu_{1} \mu_{2}}^{M}+\frac{9}{2} \Lambda_{N} \Theta^{P} D_{\alpha, P}{ }^{N} D_{Q}^{\alpha M} A_{\mu_{1} \mu_{2}}^{Q} \\
& \delta A_{\mu_{1} \mu_{2} \mu_{3}}^{\alpha}=\partial_{\left[\mu_{1}\right.} A_{\left.\mu_{2} \mu_{3}\right]}^{\alpha}+a_{\left[\mu_{1}, M\right.} A_{\left.\mu_{2} \mu_{3}\right]}^{N} D_{N}^{\alpha M}+\frac{1}{6} a_{\left[\mu_{1}, M\right.} A_{\mu_{2}, N} A_{\left.\mu_{3}\right], P} d^{M N Q} D_{Q}^{\alpha P} \\
& \quad+3 \Lambda_{M} \Theta^{M} A_{\mu_{1} \mu_{2} \mu_{3}}^{\alpha}-\frac{9}{2} \Lambda_{N} \Theta^{P} D_{\beta, P}{ }^{N} f^{\beta \gamma}{ }_{\alpha} A_{\mu_{1} \mu_{2} \mu_{3}, \gamma}
\end{aligned}
$$

where using eq. (2.30) one obtains the parameters $a_{\mu_{1} \ldots \mu_{n}, M_{n}}$ in terms of the gauge parameters as

$$
\begin{aligned}
& a_{\mu, M}=\partial_{\mu} \Lambda_{M}+15 \Theta^{N} d_{M N P} \Lambda_{\mu}^{P} \\
& a_{\mu_{1} \mu_{2}}^{M}=\partial_{\left[\mu_{1}\right.} \Lambda_{\left.\mu_{2}\right]}^{M}+\frac{27}{2} \Theta^{N} D_{N}^{\alpha M} \Lambda_{\mu_{1} \mu_{2}, \alpha}
\end{aligned}
$$

We now consider the six-dimensional case. 


\section{$6 \quad \mathrm{D}=6$}

The symmetry of the massless maximal supergravity theory in 6 dimensions [37] is $S O(5,5)$, and the bosonic sector of the theory describes 25 scalars parametrising the symmetric manifold $S O(5,5) /[S O(5) \times S O(5)]$, the metric, a 1-form in the $\mathbf{1 6}$ and a 2-form in the 10, whose field strength satisfies a self-duality condition. From $E_{11}$ this theory arises after deleting node 6 in the $E_{11}$ Dynkin diagram of fig. 1.

In this paper we will only consider the form generators of rank up to four included. For a more detailed analysis including the 5-form generators we refer to [22], whose notation we fully adopt. Using the results of [22], the analysis presented here can easily be extended to higher rank form generators. The level zero generators and the form generators up to the 4-form included are

$$
K^{\mu}{ }_{\nu}(\mathbf{1}) R^{M N}(\mathbf{4 5}) R^{\mu, \dot{\alpha}}(\overline{\mathbf{1 6}}) R^{\mu_{1} \mu_{2}, M}(\mathbf{1 0}) R^{\mu_{1} \mu_{2} \mu_{3}, \alpha}(\mathbf{1 6}) R^{\mu_{1} \mu_{2} \mu_{3} \mu_{4}, M N}
$$

where we denote in brackets the corresponding $S O(5,5)$ representations.

We review now the $S O(5,5)$ Gamma matrix conventions of [22]. We are using a Weyl basis, so that the Gamma matrices have the form

$$
\Gamma_{M, A}{ }^{B}=\left(\begin{array}{cc}
0 & \Gamma_{M, \alpha}^{\dot{\beta}} \\
\Gamma_{M, \dot{\alpha}}^{\beta} & 0
\end{array}\right)
$$

where $A=1, \ldots, 32$. They satisfy the Clifford algebra

$$
\left\{\Gamma_{M}, \Gamma_{N}\right\}=2 \eta_{M N}
$$

where $\eta_{M N}$ is the $S O(5,5)$ Minkowski metric. The charge conjugation matrix is

$$
C^{A B}=\left(\begin{array}{cc}
0 & C^{\alpha \dot{\beta}} \\
C^{\dot{\alpha} \beta} & 0
\end{array}\right),
$$

which is antisymmetric and unitary, that is

$$
C^{\alpha \dot{\beta}}=-C^{\dot{\beta} \alpha}
$$

and

$$
C_{\alpha \dot{\beta}}^{\dagger} C^{\dot{\beta} \gamma}=\delta_{\alpha}^{\gamma} \quad C_{\dot{\alpha} \beta}^{\dagger} C^{\beta \dot{\gamma}}=\delta_{\dot{\alpha}}^{\dot{\gamma}} \quad,
$$

and satisfies the property

$$
C \Gamma_{M} C^{\dagger}=-\Gamma_{M}^{T}
$$


We now review the commutation relations of $[22]$. The $S O(5,5)$ algebra is

$$
\left[R^{M N}, R^{P Q}\right]=\eta^{M P} R^{N Q}-\eta^{N P} R^{M Q}+\eta^{N Q} R^{M P}-\eta^{M Q} R^{N P}
$$

while the commutators of the $S O(5,5)$ generators with the positive level generators are

$$
\begin{aligned}
& {\left[R^{M N}, R^{\mu, \dot{\alpha}}\right]=-\frac{1}{2} \Gamma_{\dot{\beta}}^{M N} R^{\dot{\alpha}} R^{\mu, \dot{\beta}}} \\
& {\left[R^{M N}, R^{\mu_{1} \mu_{2}, P}\right]=\eta^{M P} R^{\mu_{1} \mu_{2}, N}-\eta^{N P} R^{\mu_{1} \mu_{2}, M}} \\
& {\left[R^{M N}, R^{\mu_{1} \mu_{2} \mu_{3}, \alpha}\right]=-\frac{1}{2} \Gamma_{\beta}^{M N}{ }^{\alpha} R^{\mu_{1} \mu_{2} \mu_{3}, \beta}}
\end{aligned}
$$

The commutators of the positive level generators of eq. (6.1) are

$$
\begin{aligned}
& {\left[R^{\mu_{1}, \dot{\alpha}}, R^{\mu_{2}, \dot{\beta}}\right]=\left(C \Gamma_{M}\right)^{\dot{\alpha} \dot{\beta}} R^{\mu_{1} \mu_{2}, M}} \\
& {\left[R^{\mu_{1}, \dot{\alpha}}, R^{\mu_{2} \mu_{3}, M}\right]=\Gamma_{\alpha}^{M{ }_{\alpha}^{\alpha}} R^{\mu_{1} \mu_{2} \mu_{3}, \alpha}} \\
& {\left[R^{\mu_{1} \mu_{2}, M}, R^{\mu_{3} \mu_{4}, N}\right]=R^{\mu_{1} \ldots \mu_{4}, M N}} \\
& {\left[R^{\mu_{1}, \dot{\alpha}}, R^{\mu_{2} \mu_{3} \mu_{4}, \alpha}\right]=\frac{1}{4}\left(C \Gamma_{M N}\right)^{\dot{\alpha} \alpha} R^{\mu_{1} \ldots \mu_{4}, M N}}
\end{aligned}
$$

We now consider the trombone deformations of the $E_{11,6}^{\text {local }}$ algebra. We start from the commutators of eq. (2.5) and (2.10). Using $S O(5,5)$ Fierz identities, one can show that the Jacobi identities involving two positive level generators and the momentum generator give

$$
\begin{aligned}
& {\left[R^{\mu, \dot{\alpha}}, P_{\nu}\right]=\delta_{\nu}^{\mu}\left[\Theta_{\alpha} C^{\alpha \dot{\alpha}} K-\frac{1}{5} \Theta_{\alpha}\left(C \Gamma^{M N}\right)^{\alpha \dot{\alpha}} R_{M N}\right]} \\
& {\left[R^{\mu_{1} \mu_{2}, M}, P_{\nu}\right]=-\frac{4}{5} \Theta_{\alpha} \Gamma_{\dot{\alpha}}^{M \alpha} \delta_{\nu}^{\left[\mu_{1}\right.} R^{\left.\mu_{2}\right], \dot{\alpha}}} \\
& {\left[R^{\mu_{1} \mu_{2} \mu_{3}, \alpha}, P_{\nu}\right]=\frac{6}{5} \Theta_{\beta}\left(C \Gamma_{M}\right)^{\alpha \beta} \delta_{\nu}^{\left[\mu_{1}\right.} R^{\left.\mu_{2} \mu_{3}\right], M}} \\
& {\left[R^{\mu_{1} \ldots \mu_{4}, M N}, P_{\nu}\right]=\frac{8}{5} \Theta_{\alpha}\left(\Gamma^{M N}\right)_{\beta}{ }^{\alpha} \delta_{\nu}^{\left[\mu_{1}\right.} R^{\left.\mu_{2} \mu_{3} \mu_{4}\right], \beta}}
\end{aligned}
$$

where the embedding tensor $\Theta_{\alpha}$ is in the same representation of the 1-form generator $R^{\mu, \dot{\alpha}}$ because spinor indices are raised and lowered using the matrix $C$ of eq. (6.4).

We then consider the quadratic constraints. Eq. (2.17), applied to this case, gives

$$
\Theta_{\alpha} \Theta_{\beta}\left(C \Gamma_{M}\right)^{\alpha \beta}=0
$$

which is precisely the $S O(5,5)$ pure spinor constraint of [26]. It can be shown that all the other Jacobi identities close automatically using this equation. 
To conclude this section, we determine the field strengths and gauge transformations of the fields as they result from applying the formulae of section 2 to this six-dimensional case. We get the field strengths

$$
\begin{aligned}
& F_{\mu_{1} \mu_{2}, \dot{\alpha}}=2\left[\partial_{\left[\mu_{1}\right.} A_{\left.\mu_{2}\right], \dot{\alpha}}-\frac{1}{2} \Theta_{\beta} C^{\beta \dot{\beta}} A_{\left[\mu_{1}, \dot{\beta}\right.} A_{\left.\mu_{2}\right], \dot{\alpha}}-\frac{1}{20} A_{\left[\mu_{1}, \dot{\gamma}\right.} A_{\left.\mu_{2}\right], \dot{\delta}} \Theta_{\beta}\left(C \Gamma^{M N}\right)^{\beta \dot{\gamma}} \Gamma_{M N, \dot{\alpha}} \dot{\delta}\right. \\
& \left.+\frac{4}{5} \Theta_{\alpha} \Gamma_{\dot{\alpha}}^{M \alpha} A_{\mu_{1} \mu_{2}, M}\right] \\
& F_{\mu_{1} \mu_{2} \mu_{3}, M}=3\left[\partial_{\left[\mu_{1}\right.} A_{\left.\mu_{2} \mu_{3}\right], M}+\frac{1}{2} \partial_{\left[\mu_{1}\right.} A_{\mu_{2}, \dot{\alpha}} A_{\left.\mu_{3}\right], \dot{\beta}}\left(C \Gamma_{M}\right)^{\dot{\alpha} \dot{\beta}}-\frac{6}{5} \Theta_{\beta}\left(C \Gamma_{M}\right)^{\alpha \beta} A_{\mu_{1} \mu_{2} \mu_{3}, \alpha}\right. \\
& \left.+\frac{4}{5} A_{\left[\mu_{1} \mu_{2}, N\right.} A_{\left.\mu_{3}\right], \dot{\alpha}} \Theta_{\beta} \Gamma_{\dot{\beta}}^{N \beta}\left(C \Gamma_{M}\right)^{\dot{\alpha} \dot{\beta}}-\frac{1}{60} A_{\left[\mu_{1}, \dot{\alpha}\right.} A_{\mu_{2}, \dot{\beta}} A_{\left.\mu_{3}\right], \dot{\gamma}} \Theta_{\alpha}\left(C \Gamma^{N P}\right)^{\alpha \dot{\alpha}}\left(\Gamma_{N P}\right)_{\dot{\delta}} \dot{\beta}\left(C \Gamma_{M}\right)^{\dot{\delta} \dot{\gamma}}\right] \\
& F_{\mu_{1} \mu_{2} \mu_{3} \mu_{4}, \alpha}=4\left[\partial_{\left[\mu_{1}\right.} A_{\left.\mu_{2} \mu_{3} \mu_{4}\right], \alpha}-\partial_{\left[\mu_{1}\right.} A_{\mu_{2} \mu_{3}, M} A_{\left.\mu_{4}\right], \dot{\alpha}} \Gamma_{\alpha}^{M \dot{\alpha}}\right. \\
& -\frac{1}{6} \partial_{\left[\mu_{1}\right.} A_{\mu_{2}, \dot{\alpha}} A_{\mu_{3}, \dot{\beta}} A_{\left.\mu_{4}\right], \dot{\gamma}}\left(C \Gamma_{M}\right)^{\dot{\alpha} \dot{\beta}} \Gamma_{\alpha}^{M \dot{\gamma}}-\frac{8}{5} \Theta_{\beta} \Gamma_{\alpha}^{M N \beta} A_{\mu_{1} \ldots \mu_{4}, M N} \\
& +\frac{6}{5} A_{\left[\mu_{1} \mu_{2} \mu_{3}, \beta\right.} A_{\left.\mu_{4}\right], \dot{\alpha}} \Theta_{\gamma}\left(C \Gamma_{M}\right)^{\beta \gamma} \Gamma_{\alpha}^{M \dot{\alpha}}+\frac{2}{5} A_{\left[\mu_{1} \mu_{2}, M\right.} A_{\left.\mu_{3} \mu_{4}\right], N} \Theta_{\beta} \Gamma_{\dot{\alpha}}^{M \beta} \Gamma_{\alpha}^{N \dot{\alpha}} \\
& -\frac{2}{5} A_{\left[\mu_{1} \mu_{2}, M\right.} A_{\mu_{3}, \dot{\alpha}} A_{\left.\mu_{4}\right], \dot{\beta}} \Theta_{\beta} \Gamma_{\dot{\gamma}}^{M \beta}\left(C \Gamma_{N}\right)^{\dot{\gamma} \dot{\alpha}} \Gamma_{\alpha}^{N \dot{\beta}} \\
& \left.+\frac{1}{240} A_{\left[\mu_{1}, \dot{\alpha}\right.} A_{\mu_{2}, \dot{\beta}} A_{\mu_{3}, \dot{\gamma}} A_{\left.\mu_{4}\right], \dot{\delta}} \Theta_{\beta}\left(C \Gamma^{M N}\right)^{\beta \dot{\alpha}} \Gamma_{M N, \dot{\epsilon}} \dot{\dot{\beta}}\left(C \Gamma_{P}\right)^{\dot{\epsilon} \dot{\gamma}} \Gamma_{\alpha}^{P \dot{\delta}}\right]
\end{aligned}
$$

and the gauge transformations

$$
\begin{aligned}
& \delta A_{\mu, \dot{\alpha}}=a_{\mu, \dot{\alpha}}-\frac{1}{2} a^{M N} \Gamma_{M N, \dot{\alpha}} A_{\mu, \dot{\beta}}+\Theta_{\alpha} C^{\alpha \dot{\beta}} \Lambda_{\dot{\beta}} A_{\mu, \dot{\alpha}} \\
& \delta A_{\mu_{1} \mu_{2}, M}=a_{\mu_{1} \mu_{2}, M}-\frac{1}{2}\left(C \Gamma_{M}\right)^{\dot{\alpha} \dot{\beta}} A_{\left[\mu_{1}, \dot{\alpha}\right.} a_{\left.\mu_{2}\right], \dot{\beta}}+2 a_{M}^{N} A_{\mu_{1} \mu_{2}, N}+2 \Theta_{\alpha} \Lambda_{\dot{\alpha}} C^{\alpha \dot{\alpha}} A_{\mu_{1} \mu_{2}, M} \\
& \delta A_{\mu_{1} \mu_{2} \mu_{3}, \alpha}=\partial_{\left[\mu_{1}\right.} \Lambda_{\left.\mu_{2} \mu_{3}\right], \alpha}+\Gamma_{\alpha}^{M \dot{\alpha}} A_{\left[\mu_{1} \mu_{2}, M\right.} a_{\left.\mu_{3}\right], \dot{\alpha}}-\frac{1}{3 !}\left(C \Gamma_{M}\right)^{\dot{\beta} \dot{\gamma}} \Gamma_{\alpha}^{M \dot{\alpha}} A_{\left[\mu_{1}, \dot{\alpha}\right.} A_{\mu_{2}, \dot{\beta}} a_{\left.\mu_{3}\right], \dot{\gamma}} \\
& \quad-\frac{1}{2} a^{M N} \Gamma_{M N, \alpha}{ }^{\beta} A_{\mu_{1} \mu_{2} \mu_{3}, \beta}+3 \Theta_{\beta} C^{\beta \dot{\alpha}} \Lambda_{\dot{\alpha}} A_{\mu_{1} \mu_{2} \mu_{3}, \alpha}
\end{aligned}
$$

where using eq. (2.30) we express the parameters $a_{\mu_{1} \ldots \mu_{n}, M_{n}}$ in terms of the gauge parameters as

$$
\begin{aligned}
& a_{M N}=-\frac{1}{5} \Lambda_{\dot{\alpha}} \Theta_{\alpha}\left(C \Gamma_{M N}\right)^{\alpha \dot{\alpha}} \\
& a_{\mu, \dot{\alpha}}=\partial_{\mu} \Lambda_{\dot{\alpha}}-\frac{4}{5} \Theta_{\alpha} \Gamma_{\dot{\alpha}}^{M \alpha} \Lambda_{\mu, M} \\
& a_{\mu_{1} \mu_{2}, M}=\partial_{\left[\mu_{1}\right.} \Lambda_{\left.\mu_{2}\right], M}+\frac{6}{5} \Theta_{\beta}\left(C \Gamma_{M}\right)^{\alpha \beta} \Lambda_{\mu_{1} \mu_{2}, \alpha} .
\end{aligned}
$$

This concludes the six-dimensional analysis. 


\section{$\begin{array}{ll}7 & \mathrm{D}=7\end{array}$}

The massless maximal supergravity theory in 7 dimensions [38] has a bosonic sector containing 14 scalars parametrising $S L(5, \mathbb{R}) / S O(5)$, the metric, a 1 -form in the $\overline{\mathbf{1 0}}$ and a 2-form in the $\mathbf{5}$ of $S L(5, \mathbb{R})$. This theory results from $E_{11}$ after deletion of node 7 in fig. 1. As in all other cases, we consider the level zero generators as well as the positive level generators up to rank four included. These are

$$
K_{\nu}^{\mu}(\mathbf{1}) R_{N}^{M}(\mathbf{2 4}) R^{\mu, M N}(\mathbf{1 0}) R_{M}^{\mu_{1} \mu_{2}}(\overline{\mathbf{5}}) R^{\mu_{1} \mu_{2} \mu_{3}, M} \text { (5) } R^{\mu_{1} \mu_{2} \mu_{3} \mu_{4}}{ }_{M N}(\overline{\mathbf{1 0}})
$$

where we denote in brackets the corresponding $S L(5, \mathbb{R})$ representation.

We now review the algebraic relations of [22] between the generators in eq. (7.1). The relevant commutators are those involving the generators of $S L(5, \mathbb{R})$,

$$
\begin{aligned}
& {\left[R^{M}{ }_{N}, R^{P}{ }_{Q}\right]=\delta_{N}^{P} R^{M}{ }_{Q}-\delta_{Q}^{M} R^{P}{ }_{N}} \\
& {\left[R^{M}{ }_{N}, R^{\mu, P Q}\right]=\delta_{N}^{P} R^{\mu, M Q}+\delta_{N}^{Q} R^{\mu, P M}-\frac{2}{5} \delta_{N}^{M} R^{\mu, P Q}} \\
& {\left[R^{M}{ }_{N}, R^{\mu_{1} \mu_{2}}{ }_{P}\right]=-\delta_{P}^{M} R^{\mu_{1} \mu_{2}}{ }_{N}+\frac{1}{5} \delta_{N}^{M} R^{\mu_{1} \mu_{2}}{ }_{P}}
\end{aligned}
$$

as well as those between the positive level generators

$$
\begin{aligned}
& {\left[R^{\mu_{1}, M N}, R^{\mu_{2}, P Q}\right]=\epsilon^{M N P Q R} R^{\mu_{1} \mu_{2}} R} \\
& {\left[R^{\mu_{1}, M N}, R^{\mu_{2} \mu_{3}}{ }_{P}\right]=\delta_{P}^{[M} R^{\left.\mu_{1} \mu_{2} \mu_{3}, N\right]}} \\
& {\left[R^{\mu_{1} \mu_{2}}{ }_{M}, R^{\mu_{3} \mu_{4}}{ }_{N}\right]=R^{\mu_{1} \ldots \mu_{4}}{ }_{M N}} \\
& {\left[R^{\mu_{1}, M N}, R^{\mu_{2} \mu_{3} \mu_{4}, P}\right]=\epsilon^{M N P Q R} R^{\mu_{1} \ldots \mu_{4}}{ }_{Q R} .}
\end{aligned}
$$

To prove that all the Jacobi identities close one makes use of

$$
\epsilon^{M_{1} . . M_{5}} \epsilon_{N_{1} \ldots N_{5}}=5 ! \delta_{\left[N_{1} \ldots N_{5}\right]}^{\left[M_{1} \ldots M_{5}\right]}
$$

We now consider the trombone deformations of the $E_{11,7}^{\text {local }}$ algebra as they result from applying to this $S L(5, \mathbb{R})$ case the generic commutators of eq. (2.5) and (2.10). Imposing the closure of the Jacobi identities involving two positive level generators and momentum 
one obtains

$$
\begin{aligned}
& {\left[R^{\mu, M N}, P_{\nu}\right]=\delta_{\nu}^{\mu}\left[\Theta^{M N} K-\frac{5}{3} \Theta^{[M|P|} R_{P}^{N]}\right]} \\
& {\left[R^{\mu_{1} \mu_{2}}{ }_{M}, P_{\nu}\right]=\frac{5}{12} \epsilon_{M N P Q R} \Theta^{N P} \delta_{\nu}^{\left[\mu_{1}\right.} R^{\left.\mu_{2}\right], Q R}} \\
& {\left[R^{\mu_{1} \mu_{2} \mu_{3}, M}, P_{\nu}\right]=-5 \Theta^{M N} \delta_{\nu}^{\left[\mu_{1}\right.} R^{\left.\mu_{2} \mu_{3}\right]}{ }_{N}} \\
& {\left[R^{\mu_{1} \mu_{2} \mu_{3} \mu_{4}}{ }_{M N}, P_{\nu}\right]=\frac{5}{6} \epsilon_{M N P Q R} \Theta^{P Q} \delta_{\nu}^{\left[\mu_{1}\right.} R^{\left.\mu_{2} \mu_{3} \mu_{4}\right], R}}
\end{aligned}
$$

The quadratic constraint of eq. (2.17), when applied to this case, gives

$$
\Theta^{M N} \Theta^{P Q} \epsilon_{M N P Q R}=0
$$

and one can show that this condition in enough to guarantee the closure of all the Jacobi identities.

To conclude this section, we now determine the field strengths and gauge transformations of the fields. Starting from the group element of the form of eq. (2.27) and using eqs. (2.28) and (2.29) we get the field strength of the vectors

$$
F_{\mu_{1} \mu_{2}, M N}=2\left[\partial_{\left[\mu_{1}\right.} A_{\left.\mu_{2}\right], M N}-\frac{5}{12} \Theta^{P Q} \epsilon_{M N P Q R} A_{\mu_{1} \mu_{2}}^{R}+\frac{1}{2} \Theta^{P Q} A_{\left[\mu_{1}, P Q\right.} A_{\left.\mu_{2}\right], M N}\right]
$$

the field strength of the 2-form

$$
\begin{aligned}
F_{\mu_{1} \mu_{2} \mu_{3}}^{M} & =3\left[\partial_{\left[\mu_{1}\right.} A_{\left.\mu_{2} \mu_{3}\right]}^{M}+\frac{1}{2} \epsilon^{M N P Q R} \partial_{\left[\mu_{1}\right.} A_{\mu_{2}, N P} A_{\left.\mu_{3}\right], Q R}-5 \Theta^{M N} A_{\mu_{1} \mu_{2} \mu_{3}, N}\right. \\
& \left.-\frac{5}{12} \Theta^{R S} \epsilon^{M T U P Q} \epsilon_{N R S T U} A_{\left[\mu_{1} \mu_{2}\right.}^{N} A_{\left.\mu_{3}\right], P Q}\right]
\end{aligned}
$$

and the field strength of the 3-form

$$
\begin{aligned}
F_{\mu_{1} \ldots \mu_{4}, M} & =4\left[\partial_{\left[\mu_{1}\right.} A_{\left.\mu_{2} \ldots \mu_{4}\right], M}-\partial_{\left[\mu_{1}\right.} A_{\mu_{2} \mu_{3}}^{N} A_{\left.\mu_{4}\right], N M}-\frac{1}{6} \partial_{\left[\mu_{1}\right.} A_{\mu_{2}, N P} A_{\mu_{3}, Q R} A_{\left.\mu_{4}\right], S M} \epsilon^{S N P Q R}\right. \\
& -\frac{5}{6} A_{\mu_{1} \ldots \mu_{4}}^{N P} \Theta^{Q R} \epsilon_{M N P Q R}-5 A_{\left[\mu_{1} \mu_{2} \mu_{3}, P\right.} A_{\left.\mu_{4}\right], Q M} \Theta^{P Q} \\
& \left.+\frac{5}{24} A_{\left[\mu_{1} \mu_{2}\right.}^{R} A_{\mu_{3}, N P} A_{\left.\mu_{4}\right], Q M} \Theta^{S T} \epsilon_{R S T U V} \epsilon^{U V N P Q}\right]
\end{aligned}
$$

that are covariant under the gauge transformations

$$
\begin{aligned}
& \delta A_{\mu, M N}=a_{\mu, M N}+2 a_{[M}^{P} A_{\mu,|P| N]}+\Lambda_{P Q} \Theta^{P Q} A_{\mu, M N} \\
& \delta A_{\mu_{1} \mu_{2}}^{M}=a_{\mu_{1} \mu_{2}}^{M}-\frac{1}{2} \epsilon^{M N P Q R} A_{\left[\mu_{1}, N P\right.} a_{\left.\mu_{2}\right], Q R}-a_{N}^{M} A_{\mu_{1} \mu_{2}}^{N}+2 \Lambda_{N P} \Theta^{N P} A_{\mu_{1} \mu_{2}}^{M} \\
& \delta A_{\mu_{1} \mu_{2} \mu_{3}, M}=\partial_{\left[\mu_{1}\right.} \Lambda_{\left.\mu_{2} \mu_{3}\right], M}+A_{\left[\mu_{1} \mu_{2}\right.}^{N} a_{\left.\mu_{3}\right], N M}+\frac{1}{3 !} \epsilon^{N Q R S T} A_{\left[\mu_{1}, M N\right.} A_{\mu_{2}, Q R} a_{\left.\mu_{3}\right], S T} \\
& \quad+a_{M}{ }^{N} A_{\mu_{1} \mu_{2} \mu_{3}, N}+3 \Lambda_{N P} \Theta^{N P} A_{\mu_{1} \mu_{2} \mu_{3}, M}
\end{aligned}
$$


where the parameters $a$ are given in terms of the gauge parameters as

$$
\begin{aligned}
& a_{M}^{N}=\frac{5}{3} \Lambda_{M P} \Theta^{P N} \\
& a_{\mu, M N}=\partial_{\mu} \Lambda_{M N}+\frac{5}{12} \Theta^{P Q} \epsilon_{M N P Q R} \Lambda_{\mu}^{R} \\
& a_{\mu_{1} \mu_{2}}^{M}=\partial_{\left[\mu_{1}\right.} \Lambda_{\left.\mu_{2}\right]}^{M}+5 \Theta^{M N} \Lambda_{\mu_{1} \mu_{2}, N} .
\end{aligned}
$$

\section{$8 \quad \mathrm{D}=8$}

The maximal massless eight-dimensional supergravity was derived in [39]. Its bosonic sector contains seven scalars parametrising the manifold $S L(3, \mathbb{R}) / S O(3) \times S L(2, \mathbb{R}) / S O(2)$, the metric, a vector in the $(\overline{\mathbf{3}}, \mathbf{2})$ of the internal symmetry group $S L(3, \mathbb{R}) \times S L(2, \mathbb{R})$, a 2 -form in $(\mathbf{3}, \mathbf{1})$ and an $S L(2, \mathbb{R})$ doublet of 3 -forms which satisfy self-duality conditions. Theory corresponds to the decomposition of $E_{11}$ which results from deleting node 8 in the Dynkin diagram of fig. 1 .

As in all other cases, we consider the level zero generators and the positive level form generators with up to four indices included. These are

$$
\begin{aligned}
& R^{\mu}{ }_{\nu}(\mathbf{1}, \mathbf{1}) \quad R^{i}(\mathbf{1}, \mathbf{3}) \quad R^{M}{ }_{N}(\mathbf{8}, \mathbf{1}) \quad R^{\mu, M \alpha} \quad(\mathbf{3}, \mathbf{2}) \\
& R^{\mu_{1} \mu_{2}}{ }_{M}(\overline{\mathbf{3}}, \mathbf{1}) \quad R^{\mu_{1} \mu_{2} \mu_{3}, \alpha} \quad(\mathbf{1}, \mathbf{2}) \quad R^{\mu_{1} \mu_{2} \mu_{3} \mu_{4}, M} \quad(\mathbf{3}, \mathbf{1}) \text {, }
\end{aligned}
$$

where we have denoted in brackets their corresponding $S L(3, \mathbb{R}) \times S L(2, \mathbb{R})$ representation.

We now review the undeformed algebra of [22]. The commutators involving the scalar generators are

$$
\begin{aligned}
& {\left[R^{i}, R^{j}\right]=f^{i j}{ }_{k} R^{k}} \\
& {\left[R^{M}{ }_{N}, R^{P}{ }_{Q}\right]=\delta_{N}^{P} R^{M}{ }_{Q}-\delta_{Q}^{M} R^{P}{ }_{N}} \\
& {\left[R^{i}, R^{\mu, M \alpha}\right]=D_{\beta}^{i \alpha} R^{\mu, M \beta}} \\
& {\left[R^{M}{ }_{N}, R^{\mu, P \alpha}\right]=\delta_{N}^{P} R^{\mu, M \alpha}-\frac{1}{3} \delta_{N}^{M} R^{\mu, P \alpha}}
\end{aligned}
$$

and similarly for the higher rank form generators. Here $D_{\beta}^{i \alpha}$ are the generators of $S L(2, \mathbb{R})$ satisfying

$$
\left[D^{i}, D^{j}\right]_{\beta}^{\alpha}=f_{k}^{i j} D_{\beta}^{k \alpha}
$$

and $f^{i j}{ }_{k}$ are the structure constants of $S L(2, \mathbb{R})$. We raise and lower $S L(2, \mathbb{R})$ indices using the antisymmetric metric $\epsilon^{\alpha \beta}$, that is, for a generic doublet $V^{\alpha}$,

$$
V^{\alpha}=\epsilon^{\alpha \beta} V_{\beta} \quad V_{\alpha}=V^{\beta} \epsilon_{\beta \alpha} \quad, \quad .
$$


The generators

$$
D^{i, \alpha \beta}=\epsilon^{\alpha \gamma} D_{\gamma}^{i \beta}
$$

are symmetric in $\alpha \beta$. Useful identities relating the $S L(2, \mathbb{R})$ generators are

$$
D_{i}^{\alpha \beta} D^{i, \gamma \delta}=-\frac{1}{4}\left[\epsilon^{\alpha \gamma} \epsilon^{\beta \delta}+\epsilon^{\alpha \delta} \epsilon^{\beta \gamma}\right]
$$

and

$$
D_{\beta}^{i \gamma} D_{\gamma}^{j \alpha}+D_{\beta}^{j \gamma} D_{\gamma}^{i \alpha}=\frac{1}{2} g^{i j} \delta_{\beta}^{\alpha}
$$

where $g^{i j}$ is the $S L(2, \mathbb{R})$ Killing metric. The commutators involving the non-scalar generators of eq. (8.1) are

$$
\begin{aligned}
& {\left[R^{\mu_{1}, M \alpha}, R^{\mu_{2}, N \beta}\right]=\epsilon^{\alpha \beta} \epsilon^{M N P} R^{\mu_{1} \mu_{2}}{ }_{P}} \\
& {\left[R^{\mu_{1}, M \alpha}, R^{\mu_{2} \mu_{3}}{ }_{N}\right]=\delta_{N}^{M} R^{\mu_{1} \mu_{2} \mu_{3}, \alpha}} \\
& {\left[R^{\mu_{1} \mu_{2}}{ }_{M}, R^{\mu_{3} \mu_{4}}{ }_{N}\right]=\epsilon_{M N P} R^{\mu_{1} \mu_{2} \mu_{3} \mu_{4}, P}} \\
& {\left[R^{\mu_{1}, M \alpha}, R^{\mu_{2} \mu_{3} \mu_{4}, \beta}\right]=-\epsilon^{\alpha \beta} R^{\mu_{1} \mu_{2} \mu_{3} \mu_{4}, M}}
\end{aligned} .
$$

One can show that all Jacobi identities are satisfied. This requires the use of the identities of eqs. (8.4)-(8.7), as well as the identities

$$
\epsilon^{M_{1} M_{2} M_{3}} \epsilon_{N_{1} N_{2} N_{3}}=6 \delta_{\left[N_{1} N_{2} N_{3}\right]}^{\left[M_{1} M_{2} M_{3}\right]}
$$

and

$$
\epsilon^{\alpha \beta} \epsilon_{\gamma \delta} V^{\gamma} W^{\delta}=V^{\alpha} W^{\beta}-V^{\beta} W^{\alpha},
$$

where in the last equation $V$ and $W$ are two generic $S L(2, \mathbb{R})$ doublets.

Following the general analysis of section 2, we now consider the deformations of the $E_{11,8}^{\text {local }}$ algebra as they result from applying to this case the generic commutators of eq. (2.5) and (2.10). Imposing the closure of the Jacobi identities involving two positive level generators and momentum one obtains

$$
\begin{aligned}
& {\left[R^{\mu, M \alpha}, P_{\nu}\right]=\delta_{\nu}^{\mu}\left[\Theta^{M \alpha} K-12 \Theta^{M \beta} D_{i, \beta}{ }^{\alpha} R^{i}+3 \Theta^{N \alpha} R^{M}{ }_{N}\right]} \\
& {\left[R^{\mu_{1} \mu_{2}}{ }_{M}, P_{\nu}\right]=3 \epsilon_{M N P} \epsilon_{\alpha \beta} \Theta^{N \alpha} \delta_{\nu}^{\left[\mu_{1}\right.} R^{\left.\mu_{2}\right], P \beta}} \\
& {\left[R^{\mu_{1} \mu_{2} \mu_{3}, \alpha}, P_{\nu}\right]=0} \\
& {\left[R^{\mu_{1} \mu_{2} \mu_{3} \mu_{4}, M}, P_{\nu}\right]=-6 \epsilon_{\alpha \beta} \Theta^{M \alpha} \delta_{\nu}^{\left[\mu_{1}\right.} R^{\left.\mu_{2} \mu_{3} \mu_{4}\right], \beta}}
\end{aligned}
$$


while all other Jacobi identities close provided that the eight-dimensional case of the quadratic constraint of eq. (2.17), which is

$$
\Theta^{M \alpha} \Theta^{N \beta} \epsilon_{M N P} \epsilon_{\alpha \beta}=0
$$

holds.

Finally, we determine the field strengths and gauge transformations of the fields. Starting from the group element of eq. (2.27) and using eqs. (2.28) and (2.29) we determine the field strength of the vectors

$$
\begin{aligned}
& F_{\mu_{1} \mu_{2}, M \alpha}=2\left[\partial_{\left[\mu_{1}\right.} A_{\left.\mu_{2}\right], M \alpha}-3 \Theta^{N \beta} \epsilon_{\alpha \beta} \epsilon_{M N P} A_{\mu_{1} \mu_{2}}^{P}-\frac{3}{2} \epsilon^{\beta \gamma} A_{\left[\mu_{1}, M \beta\right.} A_{\left.\mu_{2}\right], N \gamma} \Theta_{\alpha}^{N}\right. \\
& \left.\quad-3 A_{\left[\mu_{1}, M \beta\right.} A_{\left.\mu_{2}\right], N \alpha} \Theta^{N \beta}\right],
\end{aligned}
$$

the field strength of the 2-form

$$
\begin{aligned}
& F_{\mu_{1} \mu_{2} \mu_{3}}^{M}=3\left[\partial_{\left[\mu_{1}\right.} A_{\left.\mu_{2} \mu_{3}\right]}^{M}+\frac{1}{2} \epsilon^{\alpha \beta} \epsilon^{M N P} A_{\left[\mu_{1}, N \alpha\right.} \partial_{\mu_{2}} A_{\left.\mu_{3}\right], P \beta}-3 \Theta^{N \alpha} A_{\left[\mu_{1} \mu_{2}\right.}^{R} A_{\left.\mu_{3}\right], Q \alpha} \epsilon_{R N P} \epsilon^{P Q M}\right. \\
& \left.-\frac{1}{2} A_{\left[\mu_{1}, N \beta\right.} A_{\mu_{2}, P \gamma} A_{\left.\mu_{3}\right], Q \alpha} \epsilon^{\alpha \beta} \epsilon^{M P Q} \Theta^{N \gamma}\right]
\end{aligned}
$$

and the field strength of the 3 -form

$$
\begin{aligned}
& F_{\mu_{1} \ldots \mu_{4}, \alpha}=4\left[\partial_{\left[\mu_{1}\right.} A_{\left.\mu_{2} \mu_{3} \mu_{4}\right], \alpha}+A_{\left[\mu_{1}, M \alpha\right.} \partial_{\mu_{2}} A_{\left.\mu_{3} \mu_{4}\right]}^{M}+\frac{1}{3 !} \epsilon^{\beta \gamma} \epsilon^{M N P} A_{\left[\mu_{1}, M \alpha\right.} A_{\mu_{2}, N \beta} \partial_{\mu_{3}} A_{\left.\mu_{4}\right], P \gamma}\right. \\
& -6 A_{\mu_{1} \ldots \mu_{4}, M} \Theta^{M \beta} \epsilon_{\alpha \beta}-\frac{3}{2} A_{\left[\mu_{1} \mu_{2}\right.}^{M} A_{\mu_{3}, N \beta} A_{\left.\mu_{4}\right], P \alpha} \Theta^{Q \beta} \epsilon_{M Q R} \epsilon^{R N P} \\
& \left.\quad-\frac{1}{8} A_{\left[\mu_{1}, N \gamma\right.} A_{\mu_{2}, P \delta} A_{\mu_{3}, Q \beta} A_{\left.\mu_{4}\right], M \alpha} \epsilon^{\beta \gamma} \epsilon^{M P Q} \Theta^{N \delta}\right]
\end{aligned}
$$

These field strengths transform covariantly under the gauge transformations

$$
\begin{aligned}
& \delta A_{\mu, M \alpha}=a_{\mu, M \alpha}+a_{M}^{N} A_{\mu, N \alpha}-\frac{1}{3} a_{N}{ }^{N} A_{\mu, M \alpha}+a_{i} D_{\alpha}^{i \beta} A_{\mu, M \beta}+\Lambda_{N \beta} \Theta^{N \beta} A_{\mu, M \alpha} \\
& \delta A_{\mu_{1} \mu_{2}}^{M}=a_{\mu_{1} \mu_{2}}^{M}-\frac{1}{2} \epsilon^{\alpha \beta} \epsilon^{M N P} A_{\left[\mu_{1}, N \alpha\right.} a_{\left.\mu_{2}\right], P \beta}-a_{N}{ }^{M} A_{\mu_{1} \mu_{2}}^{N}+\frac{1}{3} a_{N}{ }^{N} A_{\mu_{1} \mu_{2}}^{M}+2 \Lambda_{N \alpha} \Theta^{N \alpha} A_{\mu_{1} \mu_{2}}^{M} \\
& \delta A_{\mu_{1} \mu_{2} \mu_{3}, \alpha}=\partial_{\left[\mu_{1}\right.} \Lambda_{\left.\mu_{2} \mu_{3}\right], \alpha}+A_{\left[\mu_{1} \mu_{2}\right.}^{M} a_{\left.\mu_{3}\right], M \alpha}-\frac{1}{3 !} \epsilon^{M N P} \epsilon^{\beta \gamma} A_{\left[\mu_{1}, M \alpha\right.} A_{\mu_{2} N \beta} a_{\left.\mu_{3}\right], P \gamma} \\
& \quad+a_{i} D_{\alpha}^{i \beta} A_{\mu_{1} \mu_{2} \mu_{3}, \beta}+3 \Lambda_{M \beta} \Theta^{M \beta} A_{\mu_{1} \mu_{2} \mu_{3}, \alpha}
\end{aligned}
$$

where the parameters $a$ are given in terms of the gauge parameters $\Lambda$ as

$$
\begin{aligned}
& a_{M}^{N}=3 \Lambda_{M \alpha} \Theta^{N \alpha} \\
& a^{i}=-12 \Theta^{M \beta} D_{\alpha}^{i \beta} \Lambda_{M \alpha} \\
& a_{\mu, M \alpha}=\partial_{\mu} \Lambda_{M \alpha}-3 \epsilon_{M N P} \epsilon_{\alpha \beta} \Lambda_{\mu}^{N} \Theta^{P \beta} \\
& a_{\mu_{1} \mu_{2}}^{M}=\partial_{\left[\mu_{1}\right.} \Lambda_{\left.\mu_{2}\right]}^{M} .
\end{aligned}
$$


Using the formulae given in this paper and in [22], the reader can easily determine the field strengths and gauge transformations for the remaining fields.

\section{$9 \quad \mathrm{D}=9$}

The internal symmetry of the maximal massless nine-dimensional supergravity theory is $\mathbb{R}^{+} \times S L(2, \mathbb{R})$. The bosonic sector of the theory contains the metric, three scalars, a doublet and a singlet of vectors, a doublet of 2 -forms and a 3 -form. The decomposition of $E_{11}$ appropriate to the nine-dimensional theory corresponds to the deletion of nodes 9 and 11 in the Dynkin diagram of fig. 1. The level zero generators and the positive level form generators of rank at most four are

$$
K_{\nu}^{\mu} \quad R \quad R^{i} \quad R^{\mu} \quad R^{\mu, \alpha} \quad R^{\mu_{1} \mu_{2}, \alpha} \quad R^{\mu_{1} \mu_{2} \mu_{3}} \quad R^{\mu_{1} \mu_{2} \mu_{3} \mu_{4}}
$$

where as in the previous section $\alpha$ denotes the $S L(2, \mathbb{R})$ doublet and $i$ the $S L(2, \mathbb{R})$ triplet.

The non-trivial commutators involving the scalars and the positive level generators in eq. (9.1) are [22]

$$
\begin{aligned}
& {\left[R, R^{\mu}\right]=-R^{\mu}} \\
& {\left[R, R^{\mu, \alpha}\right]=R^{\mu, \alpha}} \\
& {\left[R^{i}, R^{\mu, \alpha}\right]=D_{\beta}^{i \alpha} R^{\mu, \beta}} \\
& {\left[R^{i}, R^{\mu_{1} \mu_{2}, \alpha}\right]=D_{\beta}^{i \alpha} R^{\mu_{1} \mu_{2}, \beta}} \\
& {\left[R^{\mu_{1}}, R^{\mu_{2}, \alpha}\right]=-R^{\mu_{1} \mu_{2}, \alpha}} \\
& {\left[R, R^{\mu_{1} \mu_{2} \mu_{3}}\right]=R^{\mu_{1} \mu_{2} \mu_{3}}} \\
& {\left[R^{\mu_{1}, \alpha}, R^{\mu_{2} \mu_{3}, \beta}\right]=\epsilon^{\alpha \beta} R^{\mu_{1} \mu_{2} \mu_{3}}} \\
& {\left[R^{\mu_{1} \mu_{2}, \alpha}, R^{\mu_{3} \mu_{4}, \beta}\right]=\epsilon^{\alpha \beta} R^{\mu_{1} \mu_{2} \mu_{3} \mu_{4}}} \\
& {\left[R^{\mu_{1}}, R^{\mu_{2} \mu_{3} \mu_{4}}\right]=-R^{\mu_{1} \mu_{2} \mu_{3} \mu_{4}} .}
\end{aligned}
$$

We now consider the deformations of the $E_{11,9}^{\text {local }}$ algebra as they result from applying to this case the generic commutators of eq. (2.5) and (2.10). Imposing the closure of the 
Jacobi identities involving two positive level generators and momentum one obtains

$$
\begin{aligned}
& {\left[R^{\mu}, P_{\nu}\right]=\delta_{\nu}^{\mu}[\Theta K+\Theta R]} \\
& {\left[R^{\mu, \alpha}, P_{\nu}\right]=\delta_{\nu}^{\mu}\left[\Theta^{\alpha} K-4 \Theta^{\beta} D_{i, \beta}{ }^{\alpha} R^{i}\right]} \\
& {\left[R^{\mu_{1} \mu_{2}, \alpha}, P_{\nu}\right]=-2 \Theta \delta_{\nu}^{\left[\mu_{1}\right.} R^{\left.\mu_{2}\right], \alpha}-\Theta^{\alpha} \delta_{\nu}^{\left[\mu_{1}\right.} R^{\left.\mu_{2}\right]}} \\
& {\left[R^{\mu_{1} \mu_{2} \mu_{3}}, P_{\nu}\right]=3 \epsilon_{\alpha \beta} \Theta^{\alpha} \delta_{\nu}^{\left[\mu_{1}\right.} R^{\left.\mu_{2} \mu_{3}\right], \beta}} \\
& {\left[R^{\mu_{1} \mu_{2} \mu_{3} \mu_{4}}, P_{\nu}\right]=-4 \Theta \delta_{\nu}^{\left[\mu_{1}\right.} R^{\left.\mu_{2} \mu_{3} \mu_{4}\right]}}
\end{aligned}
$$

where we are considering the two embedding tensors $\Theta$ and $\Theta^{\alpha}$. The only quadratic constraint that arises from imposing the closure of all the other Jacobi identities is

$$
\Theta \Theta^{\alpha}=0
$$

This constraint simply means that the two embedding tensors can not be included at the same time. It is important that the constraint $\Theta^{\alpha} \Theta^{\beta} D_{i, \alpha \beta}=0$ does not arise. Indeed, the symmetric product of two $S L(2, \mathbb{R})$ doublets is the triplet, and therefore this constraint would imply that the doublet $\Theta^{\alpha}$ should vanish identically.

In this nine-dimensional case it is also rather simple to check that these deformations are not compatible with the deformations of ref. [22] corresponding to the standard gaugings of the maximal nine-dimensional supergravity. This is exactly what happens also in the IIA case of [27], in which the trombone deformation associated to the gauged IIA theory of $[23,24]$ is not compatible with the Romans deformation.

We finally determine the field strengths and gauge transformations of the fields. Starting from the group element of eq. (2.27) and using eqs. (2.28) and (2.29) we determine the field strength of the vector, the 2 -form and the 3 -form. We consider for compactness of notation both embedding tensors in the same equations, although we know that because of eq. (9.4) they can not be simultaneously non-vanishing. The field strengths of the 1-forms are

$$
\begin{aligned}
& F_{\mu_{1} \mu_{2}}=2\left[\partial_{\left[\mu_{1}\right.} A_{\left.\mu_{2}\right]}+A_{\mu_{1} \mu_{2}, \alpha} \Theta^{\alpha}-A_{\left[\mu_{1}, \alpha\right.} A_{\left.\mu_{2}\right]} \Theta^{\alpha}\right] \\
& F_{\mu_{1} \mu_{2}, \alpha}=2\left[\partial_{\left[\mu_{1}\right.} A_{\left.\mu_{2}\right], \alpha}+2 A_{\mu_{1} \mu_{2}, \alpha} \Theta-\frac{1}{2} A_{\left[\mu_{1}, \beta\right.} A_{\left.\mu_{2}\right], \alpha} \Theta^{\beta}-\frac{1}{2} \epsilon^{\beta \gamma} A_{\left[\mu_{1}, \beta\right.} A_{\left.\mu_{2}\right], \gamma} \Theta_{\alpha}\right. \\
& \left.\quad+\frac{1}{2} A_{\left[\mu_{1}, \alpha\right.} A_{\left.\mu_{2}\right], \beta} \Theta^{\beta}\right]
\end{aligned}
$$


the field-strength of the 2-form is

$$
\begin{aligned}
& F_{\mu_{1} \mu_{2} \mu_{3}, \alpha}=3\left[\partial_{\left[\mu_{1}\right.} A_{\left.\mu_{2} \mu_{3}\right], \alpha}-A_{\left[\mu_{1}\right.} \partial_{\mu_{2}} A_{\left.\mu_{3}\right], \alpha}+3 \epsilon_{\alpha \beta} \Theta^{\beta} A_{\mu_{1} \mu_{2} \mu_{3}}-A_{\left[\mu_{1} \mu_{2}, \beta\right.} A_{\left.\mu_{3}\right], \alpha} \Theta^{\beta}\right. \\
& \left.-2 A_{\left[\mu_{1} \mu_{2}, \alpha\right.} A_{\left.\mu_{3}\right]} \Theta+A_{\left[\mu_{1}, \beta\right.} A_{\mu_{2}, \alpha} A_{\left.\mu_{3}\right]} \Theta^{\beta}+\frac{1}{2} \epsilon^{\beta \gamma} A_{\left[\mu_{1}, \beta\right.} A_{\mu_{2}, \gamma} A_{\left.\mu_{3}\right]} \Theta_{\alpha}\right]
\end{aligned}
$$

and the field strength of the 3 -form is

$$
\begin{aligned}
& F_{\mu_{1} \ldots \mu_{4}}=4\left[\partial_{\left[\mu_{1}\right.} A_{\left.\mu_{2} \ldots \mu_{4}\right]}+\epsilon^{\alpha \beta} A_{\left[\mu_{1}, \alpha\right.} \partial_{\mu_{2}} A_{\left.\mu_{3} \mu_{4}\right], \beta}+4 \Theta A_{\mu_{1} \ldots \mu_{4}}+3 \Theta^{\alpha} A_{\left[\mu_{1} \mu_{2} \mu_{3}\right.} A_{\left.\mu_{4}\right], \alpha}\right. \\
& \left.\quad-\frac{1}{2} A_{\left[\mu_{1} \mu_{2}, \alpha\right.} A_{\mu_{3}, \beta} A_{\left.\mu_{4}\right] \gamma} \Theta^{\alpha} \epsilon^{\beta \gamma}\right] .
\end{aligned}
$$

These field strengths transform covariantly under the gauge transformations

$$
\begin{aligned}
& \delta A_{\mu}=a_{\mu}+\Lambda_{\alpha} \Theta^{\alpha} A_{\mu} \\
& \delta A_{\mu, \alpha}=a_{\mu, \alpha}+2 \Lambda \Theta A_{\mu, \alpha}+\Lambda_{\beta} \Theta^{\beta} A_{\mu, \alpha}+a_{i} D_{\alpha}^{i \beta} A_{\mu, \beta} \\
& \delta A_{\mu_{1} \mu_{2}, \alpha}=a_{\mu_{1} \mu_{2}, \alpha}+A_{\left[\mu_{1}, \alpha\right.} a_{\left.\mu_{2}\right]}+2 \Lambda \Theta A_{\mu_{1} \mu_{2}, \alpha}+2 \Lambda_{\beta} \Theta^{\beta} A_{\mu_{1} \mu_{2}, \alpha}+a_{i} D_{\alpha}^{i \beta} A_{\mu_{1} \mu_{2}, \beta} \\
& \delta A_{\mu_{1} \mu_{2} \mu_{3}}=\partial_{\left[\mu_{1}\right.} \Lambda_{\left.\mu_{2} \mu_{3}\right]}-\epsilon^{\alpha \beta} A_{\left[\mu_{1} \mu_{2}, \alpha\right.} a_{\left.\mu_{3}\right], \beta}+\frac{1}{2} \epsilon^{\alpha \beta} A_{\left[\mu_{1}, \alpha\right.} A_{\mu_{2}, \beta} a_{\left.\mu_{3}\right]} \\
& \quad+4 \Lambda \Theta A_{\mu_{1} \mu_{2} \mu_{3}}+3 \Lambda_{\alpha} \Theta^{\alpha} A_{\mu_{1} \mu_{2} \mu_{3}}
\end{aligned}
$$

where the parameters $a$ are expressed in terms of the gauge parameters $\Lambda$ as

$$
\begin{aligned}
& a^{i}=-4 \Lambda_{\alpha} \Theta^{\beta} D_{\beta}^{i \alpha} \\
& a_{\mu}=\partial_{\mu} \Lambda-\Lambda_{\mu, \alpha} \Theta^{\alpha} \\
& a_{\mu, \alpha}=\partial_{\mu} \Lambda_{\alpha}-2 \Lambda_{\mu, \alpha} \Theta \\
& a_{\mu_{1} \mu_{2}, \alpha}=\partial_{\left[\mu_{1}\right.} \Lambda_{\left.\mu_{2}\right], \alpha}-3 \epsilon_{\alpha \beta} \Theta^{\beta} \Lambda_{\mu_{1} \mu_{2}}
\end{aligned}
$$

The maximal supergravity theory in nine dimensions corresponding to the gauging of the trombone symmetry was derived in [25]. This nine-dimensional case concludes the analysis carried out in this paper.

\section{Conclusions}

In [27] a new type of deformation of the local $E_{11}$ algebra corresponding to the IIA theory was derived, and it was shown that it describes the gauged IIA theory of [23, 24]. In this paper we have generalised these results to all dimensions, determining all possible

trombone deformations of the local $E_{11}$ algebra in $D$ dimensions. With respect to the 
deformations analysed in [22], these deformations not only involve the generators of the internal symmetry, but also the trace of the $G L(D, \mathbb{R})$ generators. The deformations are parametrised by a constant quantity in the same representation $\mathbf{R}_{\mathbf{1}}$ of $E_{11-D}$ as the 1 form generators. This quantity is identified with the embedding tensor introduced in [26] to describe the gauge algebra of the maximal supergravity theories in which the scaling symmetry is gauged in dimension from three to six. We have determined the quadratic constraints resulting from closure of the Jacobi identities, which coincide with the quadratic constraints of [26]. We have also determines the field strengths and gauge transformations for the 1-forms, 2-forms and 3-forms of all theories.

The deformed algebra can naturally be extended to include higher rank form generators, and we expect the field equations to arise as duality relations between the corresponding field strengths. It is important to observe, though, that the $D-1$-form generators that are present in the decomposition of $E_{11}$ corresponding to the $D$ dimensional theory are in the same representations of the embedding tensors corresponding to the gauging of a subgroup of the internal symmetry $[19,20]$. Therefore, there is no form generator in the spectrum associated to this trombone embedding tensor. As already discussed in section 2 , in $[26]$ it was conjectured that the generators in the representation $G L(D, \mathbb{R})$ of mixed symmetry that we denote by $(1, D-2)$, that is the generators $R^{\mu, \nu_{1} \ldots \nu_{D-2}, M_{1}}$, that are in the same $E_{11-D}$ representation as the 1 -form generators and thus as the trombone embedding tensor, might trigger these deformations. Our point of view, though, is that because the theories considered in this paper do not admit a lagrangian formulation, the fact that there are no form generators associated to these deformations is completely consistent, and we do not expect any $E_{11}$ generator associated to a non-propagating field to play a role in triggering these deformations.

In two dimensions the scaling symmetry becomes an off-shell symmetry, that is the central extension of the affine internal symmetry group $E_{9(9)}$ [40]. As a consequence, as stressed in [26], the embedding tensor associated to the trombone gauging and the one associated to the internal gauging coincide. This is completely consistent from the $E_{11}$ point of view. Indeed the embedding tensor associated to the trombone gauging belongs to the $\mathbf{R}_{\mathbf{1}}$ representation of $E_{11-D}$, that is the representation to which the 1 -form generators belong, while the embedding tensor associated to the internal gauging belongs to the $\mathbf{R}_{\mathbf{D}-\mathbf{1}}$ representation of the $D-1$ form generators $[19,20]$. In $D=2$ the 1 -forms and the $D-1$ forms coincide. The $E_{11}$ decomposition associated to the two-dimensional theory results 
from deleting node 2 in the Dynkin diagram of fig. 1. From the diagram it is manifest that the 1-form generators belong to the $E_{9}$ representation with $p_{3}=1$, where $p_{3}$ is the Dynkin index associated to node 3 in the diagram. The gaugings of the maximal supergravity theory in two dimensions using the embedding tensor formalism were derived in [41], and it would be interesting to analyse this from the $E_{11}$ perspective.

Finally, it is worth mentioning that the algebraic construction derived in this paper can be extended to other supergravity theories with less supersymmetry whose bosonic sector still admits a description in terms of a very-extended Kac-Moody algebra. In particular, all theories with 16 supercharges are described in terms of very-extended algebras of $B$ or $D$ type [42], while in [43] it was shown that all theories with eight supersymmetries whose reduction to three dimensions gives rise to scalars that parametrise symmetric manifolds correspond to non-linear realisations of very-extended Kac-Moody algebras for suitable choices of real forms. The same applies to all supergravity theories with more than 16 supercharges [43]. For all these theories we expect that the trombone gaugings are associated to deformations of the corresponding very-extended algebras as described in section 2 , hence the title of this paper.

\section{Acknowledgments}

I would like to thank the organisers of the FPUK v3.0 conference in Cambridge for creating a stimulating environment while this project was at its early stages. This work is supported by the PPARC rolling grant PP/C5071745/1, the EU Marie Curie research training network grant MRTN-CT-2004-512194 and the STFC rolling grant ST/G000/395/1. 


\section{References}

[1] E. Cremmer, B. Julia and J. Scherk, "Supergravity theory in 11 dimensions," Phys. Lett. B 76 (1978) 409.

[2] J. H. Schwarz and P. C. West, "Symmetries And Transformations Of Chiral N=2 D = 10 Supergravity," Phys. Lett. B 126 (1983) 301; P. S. Howe and P. C. West, "The Complete N=2, D = 10 Supergravity," Nucl. Phys. B 238 (1984) 181; J. H. Schwarz, "Covariant Field Equations Of Chiral N=2 D = 10 Supergravity," Nucl. Phys. B 226 (1983) 269.

[3] E. Cremmer and B. Julia, "The N=8 Supergravity Theory. 1. The Lagrangian," Phys. Lett. B 80 (1978) 48; "The SO(8) Supergravity," Nucl. Phys. B 159 (1979) 141.

[4] B. Julia, "Group Disintegrations," in Superspace and Supergravity, Eds. S.W. Hawking and M. Rocek (Cambridge Univ. Press, 1981); E. Cremmer, "Supergravities In 5 Dimensions," in Superspace and Supergravity, Eds. S.W. Hawking and M. Rocek (Cambridge Univ. Press, 1981).

[5] B. de Wit and H. Nicolai, "N=8 Supergravity With Local $\mathrm{SO}(8) \mathrm{X} \mathrm{SU}(8)$ Invariance," Phys. Lett. B 108 (1982) 285; "N=8 Supergravity," Nucl. Phys. B 208 (1982) 323.

[6] H. Nicolai and H. Samtleben, "Maximal gauged supergravity in three dimensions," Phys. Rev. Lett. 86 (2001) 1686 [arXiv:hep-th/0010076]; "Compact and noncompact gauged maximal supergravities in three dimensions," JHEP 0104 (2001) 022 [arXiv:hep-th/0103032].

[7] B. de Wit, H. Samtleben and M. Trigiante, "On Lagrangians and gaugings of maximal supergravities," Nucl. Phys. B 655 (2003) 93 [arXiv:hep-th/0212239].

[8] B. de Wit, H. Samtleben and M. Trigiante, "The maximal D $=5$ supergravities," Nucl. Phys. B 716 (2005) 215 [arXiv:hep-th/0412173].

[9] H. Samtleben and M. Weidner, "The maximal D $=7$ supergravities," Nucl. Phys. B 725 (2005) 383 [arXiv:hep-th/0506237].

[10] B. de Wit, H. Samtleben and M. Trigiante, "Magnetic charges in local field theory," JHEP 0509 (2005) 016 [arXiv:hep-th/0507289].

[11] B. de Wit, H. Samtleben and M. Trigiante, "The maximal D $=4$ supergravities," JHEP 0706 (2007) 049 [arXiv:0705.2101 [hep-th]]. 
[12] E. Bergshoeff, H. Samtleben and E. Sezgin, "The Gaugings of Maximal D=6 Supergravity," JHEP 0803 (2008) 068 [arXiv:0712.4277 [hep-th]].

[13] P. C. West, "E(11) and M theory," Class. Quant. Grav. 18 (2001) 4443 [arXiv:hepth/0104081].

[14] V. I. Ogievetsky, "Infinite-dimensional algebra of general covariance group as the closure of finite-dimensional algebras of conformal and linear groups," Lett. Nuovo Cim. 8 (1973) 988.

[15] A. B. Borisov and V. I. Ogievetsky, "Theory of dynamical affine and conformal symmetries as gravity theory of the gravitational field," Theor. Math. Phys. 21 (1975) 1179 [Teor. Mat. Fiz. 21 (1974) 329].

[16] P. C. West, "Hidden superconformal symmetry in M theory," JHEP 0008 (2000) 007 [arXiv:hep-th/0005270].

[17] A. Kleinschmidt, I. Schnakenburg and P. C. West, "Very-extended Kac-Moody algebras and their interpretation at low levels," Class. Quant. Grav. 21 (2004) 2493 [arXiv:hep-th/0309198].

[18] I. Schnakenburg and P. C. West, "Massive IIA supergravity as a non-linear realisation," Phys. Lett. B 540 (2002) 137 [arXiv:hep-th/0204207].

[19] F. Riccioni and P. C. West, "The E(11) origin of all maximal supergravities," JHEP 0707 (2007) 063 [arXiv:0705.0752 [hep-th]].

[20] E. A. Bergshoeff, I. De Baetselier and T. A. Nutma, "E(11) and the embedding tensor," JHEP 0709 (2007) 047 [arXiv:0705.1304 [hep-th]].

[21] F. Riccioni and P. West, "Local E(11)," JHEP 0904 (2009) 051 [arXiv:0902.4678 [hep-th]].

[22] F. Riccioni, D. Steele and P. West, "The E(11) origin of all maximal supergravities the hierarchy of field-strengths," JHEP 0909 (2009) 095 [arXiv:0906.1177 [hep-th]].

[23] P. S. Howe, N. D. Lambert and P. C. West, "A new massive type IIA supergravity from compactification," Phys. Lett. B 416 (1998) 303 [arXiv:hep-th/9707139].

[24] I. V. Lavrinenko, H. Lu and C. N. Pope, "Fibre bundles and generalised dimensional reductions," Class. Quant. Grav. 15 (1998) 2239 [arXiv:hep-th/9710243].

[25] E. Bergshoeff, T. de Wit, U. Gran, R. Linares and D. Roest, "(Non-)Abelian gauged supergravities in nine dimensions," JHEP 0210 (2002) 061 [arXiv:hep-th/0209205]. 
[26] A. Le Diffon and H. Samtleben, "Supergravities without an Action: Gauging the Trombone," Nucl. Phys. B 811 (2009) 1 [arXiv:0809.5180 [hep-th]].

[27] F. Riccioni, "Local E(11) and the gauging of the trombone symmetry," arXiv:1001.1316 [hep-th].

[28] E. Bergshoeff, R. Kallosh, T. Ortin, D. Roest and A. Van Proeyen, "New Formulations of D=10 Supersymmetry and D8-O8 Domain Walls," Class. Quant. Grav. 18 (2001) 3359 [arXiv:hep-th/0103233].

[29] E. A. Bergshoeff, M. de Roo, S. F. Kerstan, T. Ortin and F. Riccioni, "IIA ten-forms and the gauge algebras of maximal supergravity theories," JHEP 0607 (2006) 018 [arXiv:hep-th/0602280].

[30] F. Riccioni and P. C. West, "E(11)-extended spacetime and gauged supergravities," JHEP 0802 (2008) 039 [arXiv:0712.1795 [hep-th]].

[31] B. de Wit, H. Nicolai and H. Samtleben, "Gauged Supergravities, Tensor Hierarchies, and M-Theory," JHEP 0802 (2008) 044 [arXiv:0801.1294 [hep-th]].

[32] E. A. Bergshoeff, J. Gomis, T. A. Nutma and D. Roest, "Kac-Moody Spectrum of (Half-)Maximal Supergravities," JHEP 0802 (2008) 069 [arXiv:0711.2035 [hep-th]].

[33] F. Riccioni and P. C. West, "Dual fields and E(11)," Phys. Lett. B 645 (2007) 286 [arXiv:hep-th/0612001].

[34] N. Marcus and J. H. Schwarz, "Three-Dimensional Supergravity Theories," Nucl. Phys. B 228 (1983) 145.

[35] K. Koepsell, H. Nicolai and H. Samtleben, "On the Yangian (Y(e(8))) quantum symmetry of maximal supergravity in two dimensions," JHEP 9904 (1999) 023 [arXiv:hepth/9903111].

[36] C. Hillmann, "E7(7) invariant Lagrangian of $d=4 \mathrm{~N}=8$ supergravity," arXiv:0911.5225 [hep-th].

[37] Y. Tanii, "N=8 Supergravity In Six-Dimensions," Phys. Lett. B 145 (1984) 197.

[38] E. Sezgin and A. Salam, "Maximal Extended Supergravity Theory In SevenDimensions," Phys. Lett. B 118 (1982) 359.

[39] A. Salam and E. Sezgin, "D = 8 Supergravity," Nucl. Phys. B 258 (1985) 284. 
[40] B. Julia, "Infinite Lie Algebras In Physics," Invited talk given at Johns Hopkins Workshop on Current Problems in Particle Theory, 1981.

[41] H. Samtleben and M. Weidner, "Gauging hidden symmetries in two dimensions," JHEP 0708 (2007) 076 [arXiv:0705.2606 [hep-th]].

[42] I. Schnakenburg and P. West, "Kac-Moody symmetries of ten-dimensional nonmaximal supergravity theories," JHEP 0405 (2004) 019 [arXiv:hep-th/0401196].

[43] F. Riccioni, A. Van Proeyen and P. C. West, "Real forms of very extended KacMoody algebras and theories with eight supersymmetries," JHEP 0805 (2008) 079 [arXiv:0801.2763 [hep-th]]. 